\title{
1 The language of social touch is intuitive and quantifiable
}

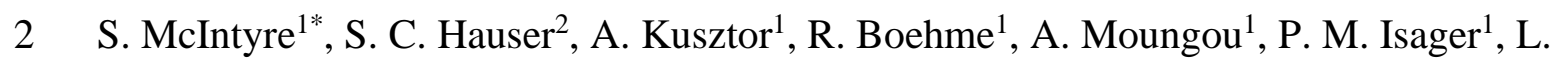

3 Homman $^{1}$, G. Novembre ${ }^{1}$, S. S. Nagi ${ }^{1}$, A. Israr ${ }^{3}$, E. A. Lumpkin ${ }^{4}$, F. Abnousi ${ }^{3}$, G. J. Gerling ${ }^{2}$, H.

4 Olausson $^{1}$

$5 \quad{ }^{1}$ Linköping University, Sweden. ${ }^{2}$ University of Virginia, USA. ${ }^{3}$ Facebook, USA. ${ }^{4}$ Columbia University, USA.

$6 *$ Correspondence to: sarah.mcintyre@liu.se

8 Abstract: Touch is a powerful communication tool, but we have a limited understanding of the

9 role played by particular physical features of interpersonal touch communication. In this study,

10 adults living in Sweden performed a task in which messages (attention, love, happiness, calming,

11 sadness and gratitude) were conveyed by a sender touching the forearm of a receiver, who

12 interpreted the messages. Two experiments $(n=32, n=20)$ showed that within close relationships,

13 receivers could identify the intuitive touch expressions of the senders, and we characterized the

14 physical features of the touches associated with successful communication. Facial expressions

15 measured with EMG varied by message, but were uncorrelated with communication

16 performance. We developed standardized touch expressions and quantified the physical features

17 with 3D hand-tracking. In two further experiments $(n=20, n=16)$, these were conveyed by trained

18 senders and were readily understood by naïve strangers. Thus, the possibility emerges of a

19 standardized, intuitively understood language of social touch. 
21 Statement of Relevance: Communicating via touch is a pre-cursor of spoken language - both in

22 an evolutionary sense, and in human infant development. This suggests the possibility of an

23 intuitive touch language with widely understood features, similar to how emoji convey facial

24 expressions. We identify a number of primitive elements of touch expressions used during

25 interpersonal communication, and describe them in terms of their physical characteristics. We

26 demonstrate that this approach can be used to compose non-verbal social messages like love,

27 attention, happiness, sadness, gratitude and calming, and that these messages are intuitively

28 understood by strangers with minimal context, and without feedback or training. 


\section{Introduction}

30 Interpersonal touch is important for social interaction, precedes language as a form of

31 communication in infants (Hertenstein, 2002), and is linked to the evolutionary development of

32 language (Dunbar, 1993). In non-human primates, touch in the form of grooming is used for

33 reconciliation after conflicts and promotes group cohesion (Dunbar, 2010). The way that adult

34 humans use interpersonal touch may be governed by this evolutionary and developmental

35 heritage. That is, there may exist neural infrastructure that is related to how the particular

36 features of interpersonal touch (e.g. tapping, stroking or holding) are used for different purposes

37 (e.g. getting someone's attention, soothing or expressing affection). However, it is currently

38 unknown if humans communicate via a common repertoire of touch gestures, or what determines

39 successful communication.

40 There are challenges to investigating inter-personal touch in humans: touch usually happens in

41 private and complex social contexts (Cekaite \& Kvist Holm, 2017), and is governed by the

42 relationship between the people involved (Suvilehto et al., 2015, 2019). Nonetheless, several

43 studies show that touch alone effectively communicates emotions such as anger, fear, disgust,

44 love, gratitude, sympathy, happiness, and sadness (Hertenstein et al., 2009; Hertenstein, Keltner,

45 et al., 2006; Hertenstein, Verkamp, et al., 2006). Indeed, touch is the preferred channel over body

46 language and facial expressions for expressing both love and sympathy (App et al., 2011).

47 Happiness, sadness, anger and disgust are also successfully communicated, despite touch not

48 being the preferred communication channel, likely because redundancy improves communication

49 success (App et al., 2011). 
50 An outstanding question is to what extent interpersonal touch strategies are universal, culturally

51 determined, or idiosyncratic. One study shows that couples are more effective at touch

52 communication than strangers (Thompson \& Hampton, 2011), suggesting that couples may

53 develop an idiosyncratic set of touch expressions, or touch language. Other studies describe the

54 physical features of social touch (Hertenstein et al., 2009; Hertenstein, Keltner, et al., 2006; Jung

55 et al., 2015; Masson \& Op de Beeck, 2018), but do not evaluate the different strategies for

56 communication effectiveness. A recent study shows that the speed of stroking and the touched

57 body location influence the touch receiver's judgments about the emotional state and intentions

58 of the person touching them, even when they are not trying to communicate a specific message

59 (Kirsch et al., 2018). These findings suggest that some physical features of interpersonal touch

60 could be reliably mapped to different messages.

61 Observing facial expressions can be a source of additional information for the receiver of a touch

62 communication (Tsalamlal et al., 2018, 2015), and facial muscle activity changes in a way that is

63 consistent with positive affect when people are gently stroked by a soft brush (Mayo et al., 2018;

64 Pawling et al., 2017; Ree et al., 2020). It is not known whether there is a link between the

65 affective state as measured by facial muscle activity and the capacity to either convey or interpret

66 messages via touch.

67 In the current study, we investigated whether it is possible to optimize touch strategies by

68 selecting specific physical features, so that touch messages can be understood broadly within one

69 cultural context, outside of a close relationship. We first identified, within close relationships, the

70 physical features of interpersonal touch (e.g. slow stroking, tapping with a finger) that were most

71 associated with success and failure to communicate cued touch messages. We also investigated

72 facial expressions that accompany touch communication, using facial electromyography 
73 (fEMG), and tested whether variations in facial expressions were related to performance on the

74 touch communication task.

75 We then developed standardized touch expressions based on the most successful touch features

76 that we identified for each message. These were delivered by trained experimenters to naïve

77 strangers, and performance on the communication task was compared to that between people in a

78 pre-existing relationship. We also present a quantitative description in terms of motion and

79 contact area characteristics for the standardized touch expressions.

\section{Methods}

81 We recruited pairs of participants with an existing close relationship (Table 1) to participate in

82 our touch communication task (Figure 1A). The participants assigned to the role of sender were

83 required to communicate a series of messages using touch alone, using any gestures they felt

84 were appropriate. The receiver then had to identify the message from a list of options. Because

85 we gave no instructions about the touch strategies to use, we refer to this as 'intuitive' touch

86 communication to contrast it with the standardized touch strategies that we later developed.

\section{Participants}

88 For the intuitive touch experiments (experiments 1 and 2), we recruited pairs of adult participants

89 with a pre-existing relationship in which they felt emotionally close and were comfortable

90 touching each other in a normal social context. For the standardized touch experiments

91 (experiments 3 and 4), participants were recruited individually. Table 1 offers a detailed

92 description of our sample and an overview of the experiments. Participants were recruited from

93 online and poster advertisement targeting the general community and the university community 
94 at Linköping University, Sweden. This is an international community, but we did not collect any

95 data on the cultural background of our participants. All participants provided informed consent,

96 and the study was conducted in accordance with the regulations of the Regional Ethics

97 Committee who approved the study. In addition to the touch communication task, participants

98 completed a number of questionnaires, detailed in the Supplemental Material. 
Table 1. Overview of experiments and participants (* indicates data presented in a separate manuscript;

$100 * *$ indicates small deviations from the intended number of trials, see text for details).

\begin{tabular}{|c|c|c|c|c|}
\hline & Experiment 1 & Experiment 2 & Experiment 3 & Experiment 4 \\
\hline Touch type & $\begin{array}{l}\text { Intuitive } \\
\text { (both roles) }\end{array}$ & $\begin{array}{l}\text { Intuitive } \\
\text { (one role) }\end{array}$ & $\begin{array}{l}\text { Standardized } \\
\text { (expert 1) }\end{array}$ & $\begin{array}{l}\text { Standardized } \\
\text { (experts } 2 \& 3 \text { ) }\end{array}$ \\
\hline Measurements & $\begin{array}{l}\text { Communication } \\
\text { task } \\
\text { Facial EMG } \\
\text { Video recording }\end{array}$ & $\begin{array}{l}\text { Communication } \\
\text { task } \\
\text { Functional MRI* }\end{array}$ & $\begin{array}{l}\text { Communication } \\
\text { task }\end{array}$ & $\begin{array}{l}\text { Communication } \\
\text { task } \\
\text { Hand-tracking }\end{array}$ \\
\hline N participants & 32 (16 pairs) & 20 (plus partners) & 20 & 16 \\
\hline $\mathbf{N}$ trials & $\begin{array}{l}960 \\
(6 \text { cues } x \\
5 \text { repeats } x \\
32 \text { participants })\end{array}$ & $\begin{array}{l}2400 \\
(6 \text { cues } x \\
20 \text { repeats } x \\
20 \text { receivers })\end{array}$ & $\begin{array}{l}1750^{* *} \\
(6 \text { cues } x \\
15 \text { repeats } x \\
20 \text { participants })\end{array}$ & $\begin{array}{l}1916^{* *} \\
(6 \text { cues } x \\
20 \text { repeats } x \\
16 \text { participants })\end{array}$ \\
\hline $\begin{array}{l}\text { Age range } \\
\text { (median) }\end{array}$ & $19-46(24)$ & $\begin{array}{l}19-32(22) \\
\text { (partners } 19-40 \\
(22))\end{array}$ & $20-40(25)$ & $20-35(24)$ \\
\hline Sex & $\begin{array}{l}15 \text { female } \\
17 \text { male }\end{array}$ & $\begin{array}{l}9 \text { female } \\
11 \text { male } \\
\text { (partners } 11 \mathrm{~F}, 9 \mathrm{M} \text { ) }\end{array}$ & $\begin{array}{l}9 \text { female } \\
11 \text { male }\end{array}$ & $\begin{array}{l}5 \text { female } \\
11 \text { male }\end{array}$ \\
\hline Relationships & $\begin{array}{l}10 \text { romantic } \\
(9 \mathrm{FM}, 1 \mathrm{MM}) \\
5 \text { friendships } \\
(2 \mathrm{FF}, 2 \mathrm{MM}, 1 \mathrm{FM}) \\
1 \text { family }(\mathrm{FM})\end{array}$ & $\begin{array}{l}9 \text { romantic } \\
(1 \mathrm{FF}, 8 \mathrm{FM}) \\
11 \text { friendships } \\
(5 \mathrm{FF}, 6 \mathrm{MM})\end{array}$ & $\begin{array}{l}20 \text { strangers } \\
(1 \text { experimenter } \\
20 \text { participants })\end{array}$ & $\begin{array}{l}16 \text { strangers } \\
(2 \text { experimenters } \\
16 \text { participants })\end{array}$ \\
\hline
\end{tabular}

102 In experiments 1,3 and 4, the target number of participants was $15-20$, based on previous

103 research (McIntyre et al., 2019) in which $95 \%$ confidence intervals of approximately $\pm 10 \%$

104 performance was achieved at the cue level. For experiment 2, the target was set at 20 
105 participants, the recommended minimal sample size for functional imaging studies (Murphy \&

106 Garavan, 2004). The number of trials per participant was based on trying to maximize the

107 number of trials while staying within an acceptable duration for the participants. This was

108 smaller for experiment 1 due to the time taken to apply facial EMG electrodes. For experiment 2,

109 we initially recruited 22 pairs, but one pair had to be excluded from the final analysis because

110 they did not follow the task instructions. Another pair ended the experiment early because the

111 person in the scanner felt claustrophobic. In experiments 3 and 4 some trials were not performed

112 due to experimenter or technical error. In experiment 3, there were 3 participants for which only

11370,72 and 78 of the intended 90 trials were obtained; and in experiment 4, there two participants

114 for which only 118 of the intended 120 trials were obtained.

\section{Intuitive touch communication task}

116 We chose six messages to cue: attention, love, calming, happiness, sadness and gratitude (see

117 Table 2 for full-sentence cues). We included a mix of touch messages and required only that

118 participants were sufficiently acquainted to be comfortable touching each other. Our goal was to

119 try to capture a variety of different interpersonal touch interactions that may occur in natural

120 settings. Some of the cues communicate the emotional state of the sender (happiness, sadness),

121 some attempt to elicit a response from the receiver (attention, calming), and some refer to an

122 existing social context (love, gratitude, calming). These are messages that people generally want

123 to communicate using touch and were adapted from those used in a previous study (McIntyre et

124 al., 2019) to reduce conceptual overlap between messages.

125 Participants were assigned roles: one as the sender, the other as the receiver. The sender's task

126 was to send messages by touching the receiver's arm. The receiver's task was to guess what the 
127 message was. The receiver sat in a chair (experiment 1) or lay in the MRI scanner (experiment 2;

128 MRI data are presented in a separate manuscript) with one arm resting passively, accessible to

129 the sender. The sender and receiver could not see each other's faces, which were obscured by a

130 curtain (experiment 1) or the MRI scanner (experiment 2). Participants were instructed to remain

131 quiet throughout the touch communication task, not talking or making any other noise such as

132 laughing or sighing. The touch communication task was guided by a custom python script using

133 Psychopy (Peirce, 2007). On each trial, the sender was presented with one of the sender cues

134 shown in Table 2. The sender was told to touch only the forearm, and that they could perform

135 any kind of touch that they felt was appropriate. After each touch was performed, the receiver

136 was presented with buttons on a screen labelled with all of the receiver response options shown

137 in Table 2, and had to select one to indicate what they thought the sender was communicating.

138 The cues were presented in a pseudo-random order, and the locations of the buttons on the screen

139 were shuffled on every trial. No feedback was provided.

140 Table 2. Cues for the intuitive touch communication task. The 'other' option was only presented to the

141 receiver, and only in experiment 1 . In experiment 2 , the receiver had 7 seconds to respond, otherwise the 
143 in the task.

\begin{tabular}{|c|c|c|}
\hline Cue words & Sender cues & Receiver choices \\
\hline attention & $\begin{array}{l}\text { You just heard about something that your } \\
\text { partner might find interesting. Try to get } \\
\text { their ATTENTION through touch. }\end{array}$ & $\begin{array}{l}\text { Your partner is trying to } \\
\text { get your ATTENTION. }\end{array}$ \\
\hline love & $\begin{array}{l}\text { Think of all the wonderful qualities that } \\
\text { your partner has, and how they enrich your } \\
\text { life. Try to express LOVE through touch. }\end{array}$ & $\begin{array}{l}\text { Your partner is trying to } \\
\text { express LOVE. }\end{array}$ \\
\hline happiness & $\begin{array}{l}\text { You have just received good news. You are } \\
\text { feeling very happy and you want to let } \\
\text { your partner know. Try to express } \\
\text { HAPPINESS through touch. }\end{array}$ & $\begin{array}{l}\text { Your partner is trying to } \\
\text { express HAPPINESS. }\end{array}$ \\
\hline sadness & $\begin{array}{l}\text { You have just received bad news. You are } \\
\text { feeling very sad and you want to let your } \\
\text { partner know. Try to express SADNESS } \\
\text { through touch. }\end{array}$ & $\begin{array}{l}\text { Your partner is trying to } \\
\text { express SADNESS. }\end{array}$ \\
\hline calming & $\begin{array}{l}\text { Your partner is feeling upset thinking } \\
\text { about a situation that cannot be changed. } \\
\text { Try to be CALMING through touch. }\end{array}$ & $\begin{array}{l}\text { Your partner is trying to be } \\
\text { CALMING. }\end{array}$ \\
\hline gratitude & $\begin{array}{l}\text { Your partner has just helped you solve a } \\
\text { problem. Try to communicate } \\
\text { GRATITUDE through touch. }\end{array}$ & $\begin{array}{l}\text { Your partner is trying to } \\
\text { communicate } \\
\text { GRATITUDE. }\end{array}$ \\
\hline $\begin{array}{l}\text { other/ } \\
\text { timeout }\end{array}$ & & $\begin{array}{l}\text { Your partner is trying to } \\
\text { communicate something } \\
\text { else. }\end{array}$ \\
\hline
\end{tabular}

145 There were some minor differences in how the task was conducted in experiments 1 and 2. In

146 experiment 1 , the sender could take as long as they wanted to perform the touch, and controlled

147 the pace of the experiment. After performing the touch communication task, the participants

148 switched roles so that the sender became the receiver and vice versa, and the touch

149 communication task was performed with the new roles. The experimental session consisted of

150 two blocks, one for initial and one for swapped roles, of 30 trials each. Additionally, each cue 
151 was also presented once at the beginning of the first block (initial roles), in which the receivers

152 could type in open-ended responses instead of being offered the forced-choice buttons. In

153 addition to the touch communication task, facial EMG responses of the participants were

154 measured, and the touches were recorded on video (details below).

155 In experiment 2, the task was conducted while the receiver was lying in an MRI scanner, and so

156 the task was modified slightly. In addition to the instructions described above, the senders were

157 told not to perform any shaking movements in order to avoid movement artifacts. Additionally,

158 they were required to perform each touch for exactly 10 seconds to facilitate analysis of the

159 fMRI data. To control the timing of the experiment, the sender heard the single word cues (e.g.

160 'attention') via headphones, followed by a countdown to 'go' and 'stop' signals to start and stop

161 touching. So that the senders had time to consider how to perform the touch, as they did in

162 experiment 1 , the senders were familiarized with the full cues (Table 1) before entering the

163 scanner. The receiver was not given the 'other' option, but if they failed to answer within 7

164 seconds, a 'timeout' response was recorded and the next trial was presented. The participants did

165 not swap roles. The experimental session consisted of two blocks of 60 trials each.

166 Standardized touch communication task

167 In the expert touch experiments, the participant was always the receiver and a trained

168 experimenter was the sender. Based on the video observations of the common physical features

169 of touch communication behavior between people in a close relationship (experiment 1), we

170 developed a set of 10-second standardized touch gestures to each of the six emotional messages

171 (Supplemental Material, Movies S1-S6). These standardized gestures were applied by trained

172 experimenters to the forearm, who received spoken cues via headphones. 
173 In experiment 3, one trained experimenter performed all the gestures (expert 1), and the

174 experimental session consisted of one block of 90 trials. In experiment 4, two different trained

175 experimenters performed the gestures (experts 2 and 3), and the experimental session consisted

176 of two blocks of 60 trials each, usually with a different expert performing the touches in the two

177 blocks. Because people tend to find touch from women more acceptable (Suvilehto et al., 2019),

178 the trained experimenters were all female.

\section{Data analysis for touch communication}

180 To evaluate performance on the touch communication task, we used F1 as the outcome measure.

181 F1 is a measure of performance which is suited to classification tasks in which there are more

182 than two categories, and is appropriate when both misses and false alarms are equally

183 undesirable. F1 is the harmonic mean of Recall and Precision, F1 = $2 \times$ ( Precision $\mathrm{x}$ Recall ) /

184 (Precision + Recall). Recall is the rate of cues correctly identified when present (also called hit

185 rate), and Precision is the rate of cues correctly identified when the receiver chose that cue. A

186 separate set of analyses were also conducted using Recall as the dependent variable, which

187 yielded similar results and patterns of significance (not reported).

188 We conducted separate analyses for each experiment, using a generalized linear mixed effects

189 model with a logit link function for F1 performance score as the dependent variable. For

190 experiment 1, we used fixed effects for cued word and roles (initial or swapped), and a random

191 intercept term for participant. For experiments 2-4, we used a fixed effect for cued word and a

192 random intercept term for participant. When comparing intuitive and standardized touch, we

193 used fixed effects for touch type (intuitive or expert) and cued word, and a random intercept term

194 for participant. To test for significance, parametric bootstrap with 1000 samples was used to

195 produce likelihood ratios for full versus reduced models. Because we did not have the same 
number of judgments for receivers in different experiments, the weight term supplied to the

197 model reflected the number of trials for each receiver-cue combination. Holm correction was

198 used for all post-hoc tests comparing overall performance and performance of individual cues

199 against chance, and for all post-hoc pairwise comparisons, to obtain family-wise $\alpha=0.05$

200 separately for each model. Analyses were performed using R (R Core Team, 2018) and the

201 package afex (Singmann \& Kellen, 2019), and figures were created using the packages ggplot2

202 (Wickham, 2016) and patchwork (Pedersen, 2017).

\section{Facial electromyography (fEMG)}

204 In experiment 1, emotional expression in the face during the touch communication task was

205 assessed by measuring corrugator and zygomatic reactivity in response to the touch associated

206 with each cue from both participants using a dual wireless EMG system (Dual Wireless EMG

207 BioNomadix Pair, Biopac, Goleta, CA, USA). The corrugator supercilii muscle, which furrows

208 the eyebrows, is active during frowning, and stimuli with negative valence increase its activity,

209 while stimuli with positive valence decrease its activity (Larsen et al., 2003). The zygomaticus

210 major muscle, which lift the cheeks and lips, is active during smiling, and is associated primarily

211 with positive affect (Larsen et al., 2003) and particularly with high arousal positive affect

212 (Fujimura et al., 2010). Facial EMG of the corrugator and zygomatic muscles was measured in

213 microvolts $(\mu \mathrm{V})$. EMG was measured over left brow and cheek and ground sensor on the

214 forehead (Fridlund \& Cacioppo, 1986) with $4 \mathrm{~mm} \mathrm{Ag/AgCl} \mathrm{electrodes.} \mathrm{Impedances} \mathrm{were} \mathrm{kept}$

215 below $20 \mathrm{k} \Omega$. EMG signals were amplified, 10-500 Hz band pass filtered, digitized at $1000 \mathrm{~Hz}$,

$21650 \mathrm{~Hz}$ band stop filtered, rectified, and integrated over $20 \mathrm{~ms}$ by using EMG100C amplifiers, and

217 MP160 Data Acquisition System and AcqKnowledge software from Biopac Systems (Goleta, 
218 CA, USA). The script guiding the touch communication task simultaneously delivered triggers to

219 the EMG data capture system via parallel port, and played an audio signal that was captured by

220 the video recording. This synchronizing signal was sent each time the sender pressed a button on

221 the keyboard to indicate that they were about to start performing the touch. Research assistants

222 then manually inspected the video recordings of every trial to find the frame following this signal

223 on which the touch actually started. We then used the time delay from the synchronization signal

224 to align the touch onset with the fEMG recording. The video recordings were made at 25 frames

225 per second, giving us a precision of $40 \mathrm{~ms}$ for identifying the onset of the touch (the audio was

226 sampled at $44.1 \mathrm{kHz}$ and the fEMG at $1 \mathrm{kHz}$, meaning the visual onset of the touch had the

227 lowest temporal resolution). The exact timing of the onset and offset for the touches was

228 determined in this way for all participants, except for two blocks that lacked video data. In this

229 case the onset of touch was instead determined by the timing of the button-press made by the

230 sender to indicate they were about to start touching.

\section{Data processing and analysis for fEMG}

232 For each trial, we extracted a $200 \mathrm{~ms}$ long baseline preceding touch-onset and a $4 \mathrm{~s}$ long

233 stimulation segment from touch-onset. This time-window was chosen based on inspection of the

234 data plotted over time (Figure S2), which shows that it is long enough for effects to stabilize,

235 while still including sufficient data from shorter trials. For the 'attention' cue, which provoked

236 the shortest touch durations, $35 \%$ of trials lasted longer than $4 \mathrm{~s}$. The baseline period was

237 between 1200 and 1000 ms before touch onset. This was because the sender's fEMG responses

238 had already started to change immediately before onset because they read the cue before starting

239 to perform the touch (Figure S2). Automatic artefact rejection was applied to the data within the

240 selected baseline and stimulus periods using a custom R script, and based on a procedure 
241 previously validated in a large sample by comparison with manual inspection (Künecke et al.,

242 2014). Data were flagged if the range within a sliding 50-ms window exceeded three times the

243 standard deviation of the participant's full dataset. Data within the selected time-window were

244 down-sampled to $100 \mathrm{~ms}$ bins, and a bin was rejected if it contained any flagged data samples.

245 Trials were included in the analysis if after artifact rejection they had at least ten bins remaining

246 (i.e. a total of $1 \mathrm{~s}$ ), including at least one from the baseline period. Out of 971 trials recorded, the

247 following number of trials remained in the analysis after artefact rejection: 939 trials for the

248 zygomaticus activity of the sender (attention: 155 love: 162, happiness: 148, calming: 161,

249 sadness: 158, gratitude: 155); 954 trials for the zygomaticus activity of the receiver (attention:

250 151, love: 158, happiness: 162, calming: 162, sadness: 161, gratitude: 160); 924 trials for the

251 corrugator activity of the sender (attention: 152, love: 160, happiness: 150, calming: 156,

252 sadness: 154, gratitude: 152) and 935 trials for the corrugator activity of the receiver (attention:

253 149, love: 160, happiness: 156, calming: 157, sadness: 158, gratitude: 155).

254 The data were $z$-scored within each participant for each muscle separately across all trials thus

255 resulting in the standardized activity of zygomaticus and corrugator for every participant. For

256 each trial, the mean $z$ score during the baseline phase was subtracted from the mean $z$ score

257 during the touch phase to obtain the difference scores, reflecting the muscle activity in response

258 to the touch relative to the baseline period preceding touch. We used a mixed linear model with

259 difference scores as the dependent variable, a fixed effect for cued word and a random intercept

260 term for participant. To test for significance, the Kenward-Roger approximation based on a

261 modified F test was used (Singmann \& Kellen, 2019).

262 For the machine learning analysis, we used a random forest classifier (Tin Kam Ho, 1995) with

2631,000 estimators (decision trees) using the Scikit-learn package (Pedregosa et al., 2011) with 
264 Python 2.7. The estimators are constructed in randomly selected subspaces defined by the input

265 features (from the EMG recordings), and the classifier automatically finds the most important

266 features for predicting the cue. As input to the classifier, six features were selected from the

267 cleaned dataset for each muscle, resulting in 12 features total for either sender or receiver. These

268 six features were chosen as described in (Jerritta et al., 2014; Picard et al., 2001) and consist of

269 the: 1) mean of the raw signal, 2) standard deviation of the raw signal, 3) mean of the absolute

270 values of the first differences of the raw signals, 4) mean of the absolute values of first

271 differences of normalized signals, 5) mean of the absolute values of second differences of raw

272 signals, and 6) mean of absolute values of second differences of normalized signals. Ten-fold

273 cross validation was performed to determine classifier accuracy and uncertainty, and to prevent

274 overfitting. Statistical analysis of classifier accuracy was performed (Scipy statistics package,

275 Python 2.7). To determine whether classification accuracy was better than random, the number

276 of correct classifications for each cued emotion was compared to a random classifier in a

277 contingency table and a $\chi^{2}$ test of independence of variables was then used to determine

278 statistical significance.

$279 \quad$ Video recording

280 In experiment 1 , the touch communication task was recorded on video so that we could capture

281 the features of touch such as duration, speed, target area, intensity and type of touch. Care was

282 taken to record a small area focused on the arm of the receiver, so as to avoid recording the

283 participants' faces. On the rare occasions when a participant's face entered the frame, this was

284 edited to obscure the face, and the original recording was destroyed. Videos were coded by three 
285 research assistants who were unaware of the cue presented to the sender. The timing of each

286 touch event was recorded, and they were coded for target location, intensity, and type.

287 The coding scheme was adapted from that reported in (Hertenstein, Keltner, et al., 2006).

288 Locations were categorized as the hand, wrist, lower forearm (distal) and upper forearm

289 (proximal). Intensity was coded as 'light' (skin indentation or arm movement minimal. E.g. slow

290 stroking, holding), 'moderate’ (moderate skin indentation or arm movement. E.g. fast stroking,

291 squeezing), or 'strong' (considerable skin indentation or arm movement. E.g. shaking). Touch

292 type was selected from a long list of descriptors: 'holding', 'pressing', 'lifting', 'interlocking',

293 'hugging', 'tossing', 'pulling' and 'pushing', 'stroking', 'squeezing', 'swinging' and 'massaging',

294 'pinching', 'rubbing', 'tapping', 'shaking' ,'patting', 'poking' ,'hitting', 'picking', 'scratching',

295 'slapping', 'tickling' and 'trembling'.

296 Data processing and analysis for video recordings

297 Video data were annotated with ELAN (2018). For two sessions (one half of the experiment for

298 each of two different pairs), recordings were not made due to equipment failure. Annotation text

299 was automatically matched in R against the key-words included in the coding scheme for touch

300 type, location, and intensity. Trials with missing data were checked for typos and other labelling

301 errors, which were manually corrected. Of the recorded sessions, 53 trials $(2.5 \%)$ were excluded

302 due to missing data, obscured touches, and rare touch events that did not fit the coding scheme.

303 The touched locations were simplified for presentation in Figure 1. The "wrist" was included in

304 the "distal arm" category, and the "whole arm" category included touches that covered at least

305 two different location categories. 
306 To determine the most effective touch strategies, the type, location, and intensity of the touches

307 were evaluated with respect to performance on the touch communication task, for every trial.

308 The combination of type, location, and intensity gave the full description of each touch action.

309 Some senders used multiple touch actions on a single trial, including in some cases swapping

310 back and forth between different touch actions. For this reason, we did not want to simply count

311 the number of times a touch action appeared, since these trials would mean that these repeated

312 touch actions would be overrepresented. Furthermore, on some trials in which senders used

313 multiple touch actions, one action appeared to be the dominant strategy, with more time spent on

314 it than other actions. For this reason, we did not want to simply count whether a touch action

315 appeared in a trial. We decided to capture how prominent a particular touch action was for a

316 given trial by using the proportion of trial time spent on it. This also accounts for differences in

317 trial duration.

318 To identify the best and worst touch actions for conveying each cued message, for each touch

319 action, we calculated the proportion of time it appeared in all correct trials minus all incorrect

320 trials for a given cue. This score was then used to rank the touch actions for each cue. We then

321 selected the top four touch actions (or all actions that appeared more in correct than incorrect

322 trials, if there were fewer than four i.e. with a positive score), and the bottom four touch actions

323 (or all with a negative score if there were fewer than four).

\section{Hand-tracking}

325 In experiment 4, the physical attributes of standardized touch gestures from two expert senders

326 were measured and characterized using a motion tracking system (Hauser et al., 2019).

327 Measurements were taken via custom software utilizing a LeapMotion camera system (Leap 
328 Motion, San Francisco, CA) to track the sender's hands and a Flock of Birds 6-DOF sensor

329 system (Trakstar and Model 800 sensors, Ascension, Shelburne, VT) to track the receiver's

330 forearm. Six contact metrics were considered: 1) velocity of the sender's hand normal to the

331 receiver's arm 2) velocity of the sender's hand tangential to the receiver's arm, 3) the total

332 contact area between the sender's hand and the receiver's skin, 4) the percentage of the sender's

333 palm area contacting the receiver's skin, 5) the number of the sender's fingers contacting the

334 receiver, and 6) the proportion of time in which the sender's palm was in contact with the

335 receiver. To compare across gestures, mean values for each contact metric were normalized

336 between 0 to 1 based on the population standard deviation.

337 Data Availability

338 Datasets are available on figshare (https://doi.org/10.6084/m9.figshare.c.5630842.v2), and

339 analysis scripts and materials are available on github

340 (https://github.com/SDAMcIntyre/touchcomm-psci). 


\section{Results}

342 Touch communication within close relationships

343 In two experiments involving pairs in close relationships, we found that the participants assigned

344 to the role of receiver were able to interpret the gestures based on touch alone, and select the

345 correct message at well above the chance rate (Figure 1), despite neither participant receiving

346 any feedback or training. Additionally, the correct response was the most popular one, for every

347 cue (Figure 1B, C \& E), although this was not true for all individuals (Figure S1). This

348 demonstrates that social touch messages are readily communicated within emotionally close

349 pairs, consistent with previous reports (Hertenstein et al., 2009; Hertenstein, Keltner, et al., 2006;

350 McIntyre et al., 2019). 
A

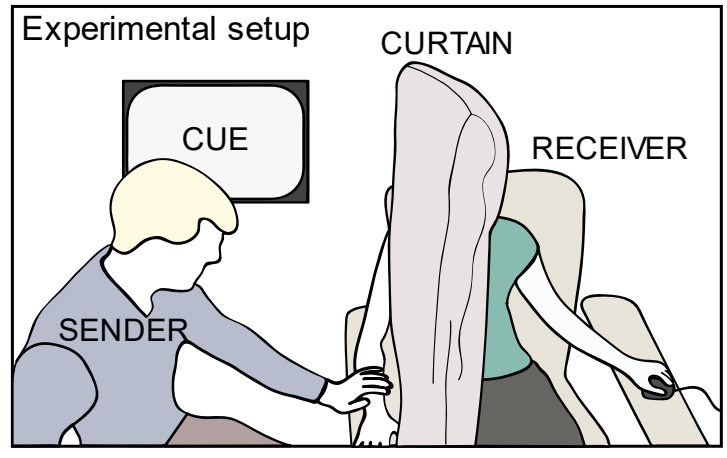

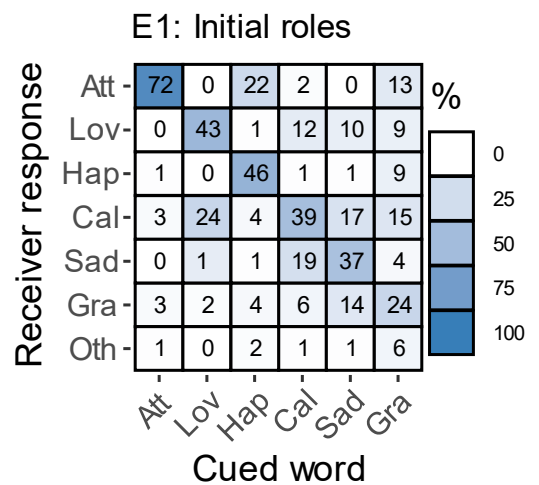

C

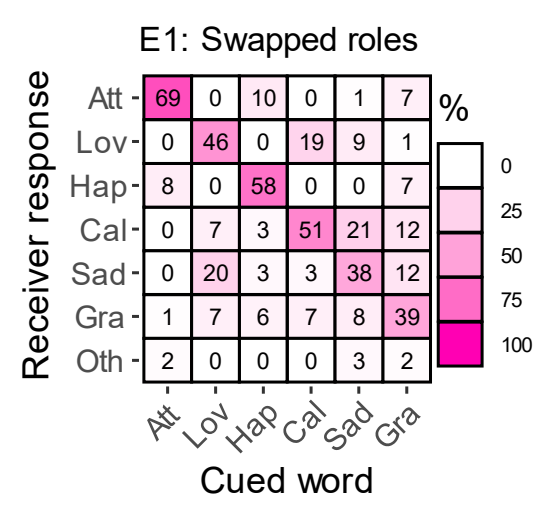

$\mathrm{E}$

Experiment 2

D

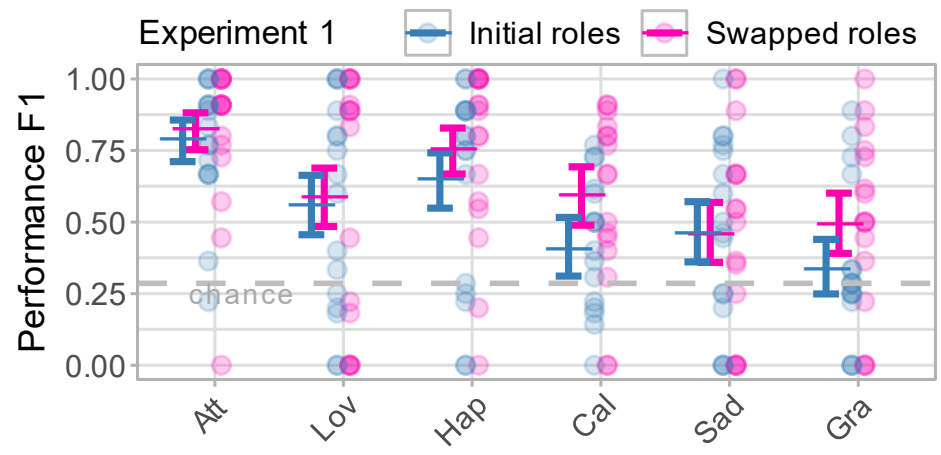

$\mathrm{F}$

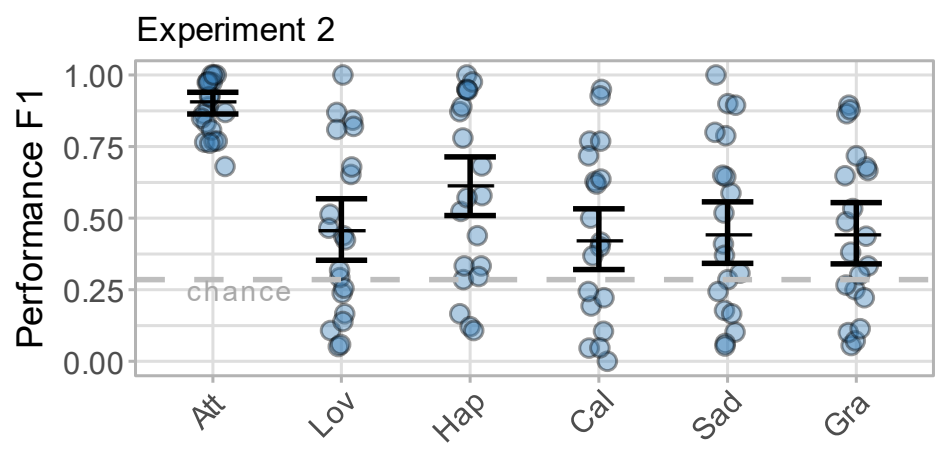

352 Figure 1. Touch communication between people in a close relationship (intuitive touch). Performance

353 was better than chance for every cue, demonstrated in two experiments. Touch cues and responses are

354 abbreviated: Att (attention), Lov (love), Hap (happiness), Cal (calming), Sad (sadness) and Gra

355 (gratitude), Oth (other), Tim (timeout). (A) Illustration of the experimental setup. The sender was

356 presented with a cue, and then touched the receiver to communicate the message. The receiver entered their responses in a separate interface. The participants could not see each others' monitors or faces. (B) Group-level confusion matrix for the communication task in the roles (sender and receiver) initially assigned; experiment 1 (E1). Total numbers of trials pooled over participants are shown inside the 
squares, and the shading gives the percentage for that cue. (C) Group-level confusion matrix after the pairs swapped roles, experiment 1 (E1). (D) F1 performance score ( $\min =0$, max $=1$; given by the harmonic mean of Recall and Precision) for experiment 1. F1 score for each pair and cue is shown as filled circles. Performance varied significantly by cue $\left(\chi^{2}(5)=432.02, p<.001\right.$, mixed effects model, see text), was significantly better after swapping roles $\left(\chi^{2}(1)=39.64, p<.001\right)$, and this effect varied with the cued word $\left(\chi^{2}(5)=24.20, p<.001\right)$. The model estimates and 95\% confidence interval are also shown. Chance-level performance $(F 1=0.29)$ is shown as a dashed line. $(\boldsymbol{E})$ Group-level confusion matrix from a second experiment, in which participants did not swap roles. $(\boldsymbol{F}) \mathrm{F} 1$ performance scores, model estimates and 95\% confidence intervals for experiment 2. Performance varied significantly by cue $\left(\chi^{2}(5)=1911.80, p<.001\right)$.

To evaluate touch communication performance, we used the F1 metric, which reflects performance that minimizes both misses and false positives. The chance rate was set at 0.29 ,

372 which is the maximum possible F1 score for a single cue when giving identical answers on all

373 trials (see Data Analysis for further details). In the first experiment (Figure 1A-D), overall

374 performance was significantly better than chance (Estimated Marginal Mean F1 score $=0.59$,

$37595 \% C I=0.49,0.68, z=18.00, p<.001$, mixed effects model, see Data Analysis for model

376 specification), as was performance for every individual cue (Figure 1D, all $p$ s $<.001$, Holm

377 correction). In this experiment only, the pairs swapped roles half-way through the testing session

378 so that the sender became the receiver and vice versa. Swapping roles significantly improved

379 identification of happiness, calming and gratitude (attention: Odds Ratio $=0.81,95 \%$ CI $=0.58$,

3801.12, z ratio $=-1.32, p=.190 ;$ love: $O R=0.89,95 \% C I=0.68,1.17, z=-0.84, p=.403$;

381 happiness: $O R=0.60,95 \% C I=0.45,0.80, z$ ratio $=-3.4, \mathrm{p}<.001$; calming: $O R=0.47,95 \% C I$

$382=0.36,0.62$, z ratio $=-5.44, p<.001 ;$ sadness: $O R=1.01,95 \% C I=0.68,1.17, z$ ratio $=-0.836$

$383 p=.403$; gratitude: $O R=0.52,95 \% C I=0.39,0.68, z$ ratio $=-4.7, p<.001 ;$ post-hoc pairwise

384 comparisons with Holm correction). 
385 In a second experiment (Figure 1E-F), the communication task was performed with the receiver

386 in an MRI scanner (data presented in a separate manuscript), and the pairs did not swap roles.

387 Similar to experiment 1 , overall performance in experiment 2 was significantly better than

388 chance $(E M M F 1$ score $=0.58,95 \% C I=0.47,0.68, z=17.8, p<.001)$, as was performance for

389 every individual cue (Figure $1 \mathrm{~F}$, all $p s<.001$, Holm correction).

390 Facial expressions accompanying touch communication

391 In order to measure facial expressions during touch communication within close relationships, in

392 experiment 1 we recorded facial muscle activity in the zygomatic (smiling) and the corrugator

393 (frowning) muscles, in both the sender and the receiver, using facial EMG (fEMG; Figure 2). For

394 the outcome measure, we used the participant level z-score of the activity measured on each

395 muscle, and took the difference in z-score measured during the first four seconds of touch

396 relative to a baseline period preceding the touch (see Methods, Figure S2). 
A. Zygomaticus

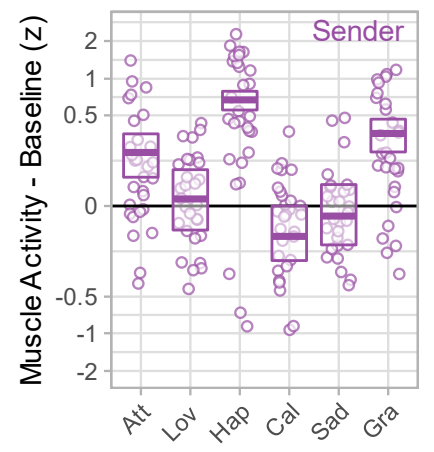

C. Sender Classifier

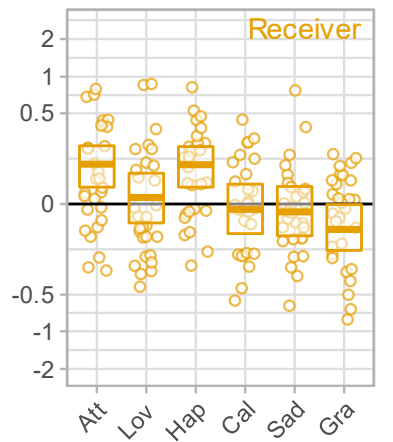

D. Receiver Classifier

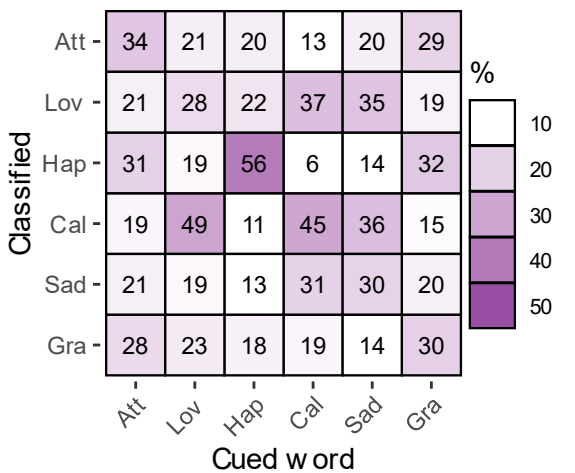

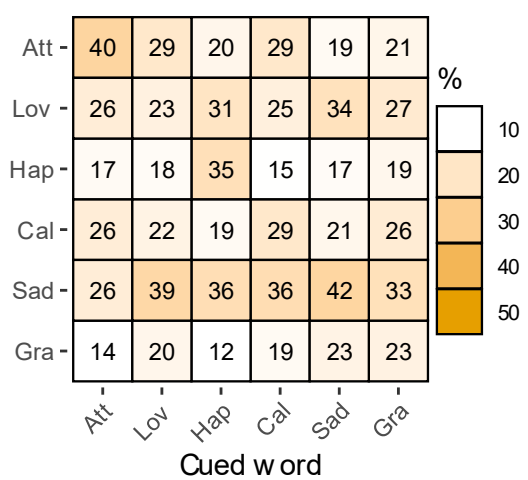

Cued word

\section{B. Corrugator}
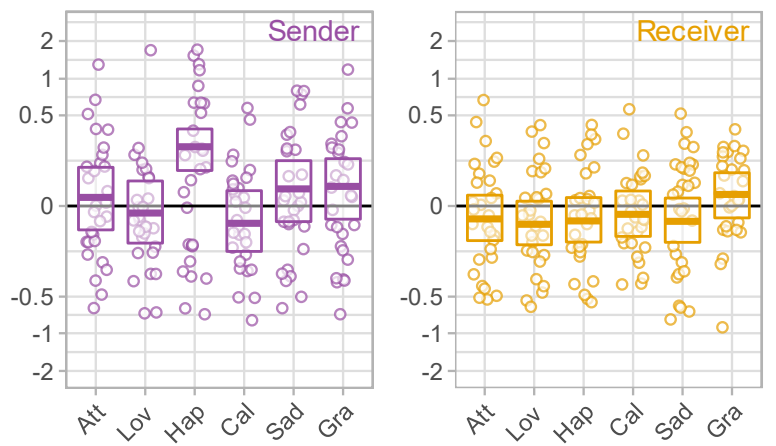

E. Correlation

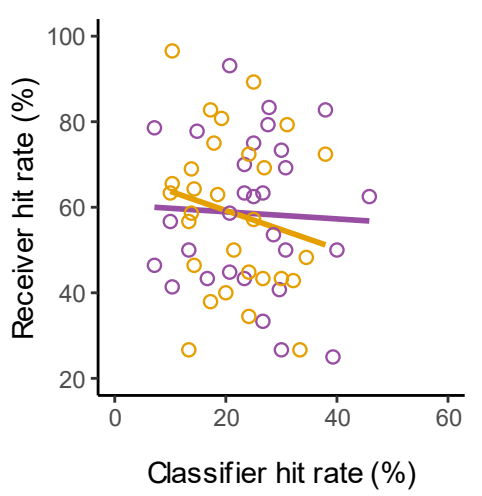

Figure 2. The role of facial expressions in touch communication. Cue abbreviations as in Figure 1. (A)

399 Mean facial EMG activity of the zygomaticus muscle during the first 4 seconds of touch during the touch

400 communication task, as participant-level z-scores during the touch, relative to baseline (y axis =0). Data

401 from a total of 939 (sender) and 954 (receiver) trials after artifact rejection (see Methods). The y-axis is

402 on a pseudo log scale to emphasize differences between the different cues rather than individual data

403 points. Circles show the average for each participant-cue combination. Cross-bars give the estimated

404 marginal mean and 95\% CI for each participant role and cue. (B) Activity of the corrugator muscle,

405 format as in (A). Data from a total of 924 (sender) and 935 (receiver) trials after artifact rejection. (C)

406 Performance of a random forest classifier on the facial muscle activity (data from both muscles, see

407 Methods) of the sender to predict the cue. (D) Performance of a random forest classifier on the facial

408 muscle activity of the receiver to predict the cue. $(\boldsymbol{E})$ The receivers' accuracy in interpreting the messages

409 was not significantly positively correlated with either classifier's performance.

410 Although the senders and receivers could not see each other's faces (Figure 1A), we found that

411 both the senders' zygomatic and corrugator activity, and the receivers' zygomatic activity varied

412 according to the cued message that was being communicated through touch. 
413 Mixed-effects models (see Data Analysis for full specification) revealed that the senders'

414 zygomatic activity during touch was significantly greater than baseline $(\mathrm{EMM}=0.19,95 \% \mathrm{CI}=$

$4150.10,0.28, \mathrm{t}(28)=4.13, \mathrm{p}<.001)$, and varied with the cue $(\mathrm{F}(5,906)=49.69, \mathrm{p}<.001$, model

416 estimates and CIs shown as crossbars, Figure 2A). The receivers' zygomatic activity during

417 touch was not overall significantly greater than baseline $(\mathrm{EMM}=0.03,95 \% \mathrm{CI}=-0.03,0.10$,

$418 \mathrm{t}(28)=1.02, \mathrm{p}=.316)$, but did vary significantly with the cue $(\mathrm{F}(5,920)=7.04, \mathrm{p}<.001$, Figure

$4192 \mathrm{~A})$.

420 The senders' overall corrugator activity was not significantly different during touch compared to 421 baseline $(\mathrm{EMM}=0.06,95 \% \mathrm{CI}=-0.04,0.157, \mathrm{t}(28)=1.14, \mathrm{p}=.264)$, but did vary significantly 422 with cue $(\mathrm{F}(5,891)=8.58, \mathrm{p}<.001$, Figure $2 \mathrm{~B})$. The receivers overall corrugator activity was not 423 significantly different during touch compared to baseline $(\mathrm{EMM}=-0.04,95 \% \mathrm{CI}=-0.10,0.03$, $424 \mathrm{t}(28)=-1.15, \mathrm{p}=.262)$, and nor did it vary with cue $(\mathrm{F}(5,901)=1.50, \mathrm{p}=.188$, Figure $2 \mathrm{~B})$.

425 We predicted that if the variations in the senders' facial expressions were related to their capacity 426 to convey social messages, the facial muscle activity of the sender should be related to the 427 successful communication of messages. Similarly, if the variations in the receivers' facial 428 expressions reflected their ability to decode the touch messages, their facial muscle activity 429 would also be related to performance on the communication task. To test this, we applied 430 machine learning methods to train random forest classifiers on the facial muscle activity of the 431 senders and the receivers separately, to predict which message was cued (see Methods). When 432 the classifier was trained on either the senders' or the receivers' facial muscle activity, it could 433 predict the cued message significantly better than chance (chance hit rate 16.7\%; sender: $24.8 \%$ $434 \pm 5.9 \%$ standard deviation across the 10 -fold cross validation procedure; $\mathrm{p}<.001, \mathrm{n}=914$ trials 435 with data available from both muscles, Figure $2 \mathrm{C}$; receiver: $21.0 \% \pm 4.4 \% \mathrm{SD} ; \mathrm{p}<.05, \mathrm{n}=931$ 
436 trials, Figure 2D). Contrary to our prediction, neither classifier's performance significantly

437 correlated with performance on the touch communication task (sender: $\mathrm{r}=-0.05,95 \% \mathrm{CI}=-$

$4380.41,0.33, \mathrm{t}(27)=-0.24, \mathrm{p}=0.8153$; receiver: $\mathrm{r}=-0.19,95 \% \mathrm{CI}=-0.52,0.19, \mathrm{t}(27)=-1.02, \mathrm{p}=$

439 0.3166; including only those individuals with classifier data for at least 10 trials; Figure 2E).

\section{Development of standardized touch gestures}

441 To characterize the intuitive touch gestures, we video-recorded the touch communication task in

442 the same experiment in which we recorded fEMG activity (experiment 1). The touching activity

443 was coded by research assistants for location, intensity, and type of touch from a list of

444 descriptors adapted from (Hertenstein, Keltner, et al., 2006). We found that successful strategies

445 for touch communication in a close relationship shared common features across senders (Figure

446 3). For attention, the best features were moderate tapping and shaking. For calming it was light

447 holding at multiple locations on the arm, as well as moderately stroking the whole or lightly

448 stroking part of the arm. Gratitude was associated with light stroking or holding, or squeezing the

449 arm. Happiness was best conveyed by light tapping across the whole arm. Love was successfully

450 signaled by light stroking across the whole arm. Successful communication of sadness was most

451 strongly associated with lightly holding or stroking one part of the arm. Touching the hand and

452 using high intensity were generally unsuccessful strategies. 


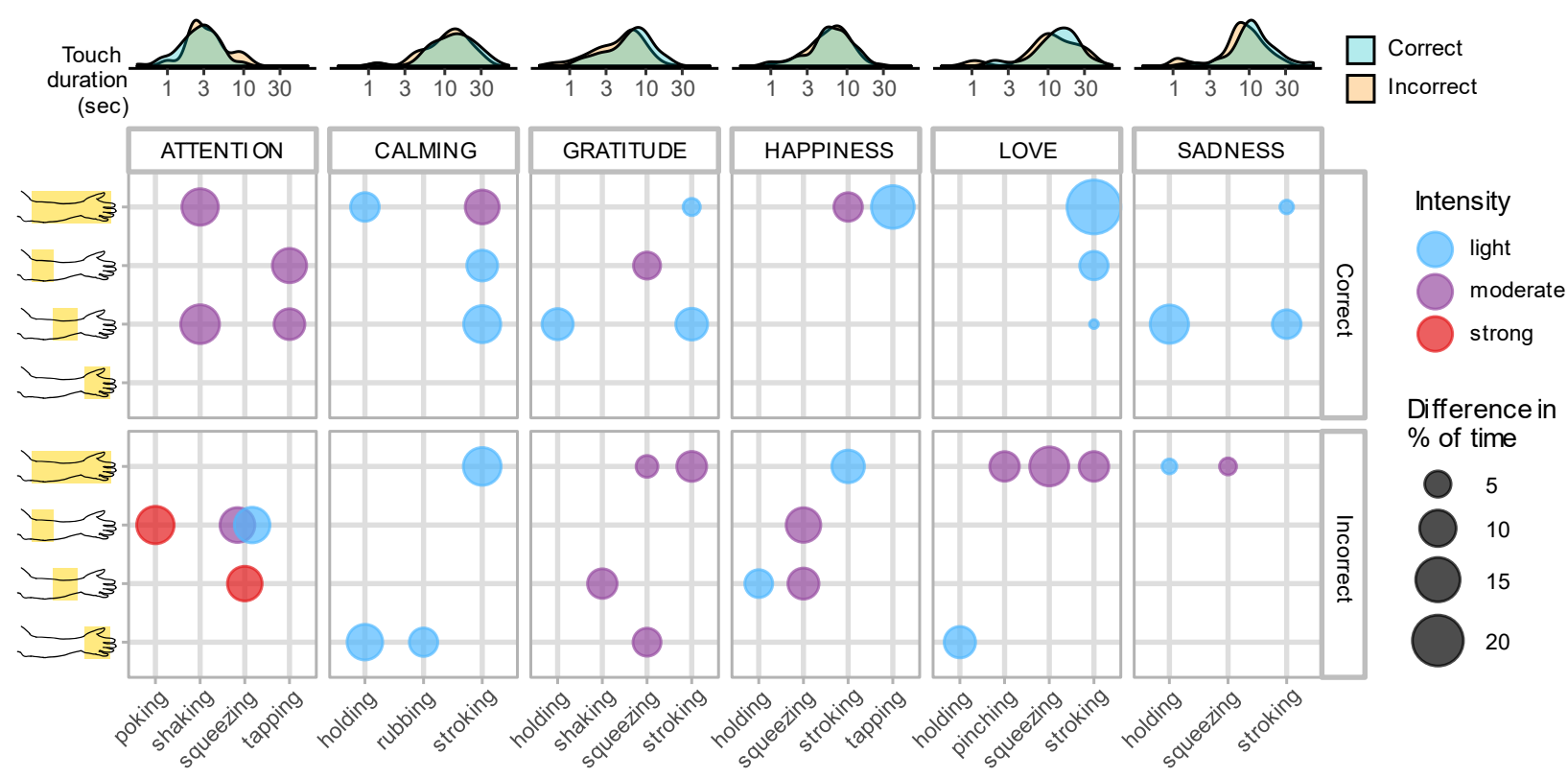

Figure 3. Location, intensity, and touch descriptors that best discriminated correct and incorrect interpretation of the messages. Density plots of the touch durations for each cue are shown above the panels. Because the overall touch duration for different cues varied, time for each combination of features was calculated as a percentage of the total touching time for each cue (see Methods for more details). The top panels show the best combinations of touch features, up to four for each cue, that were observed most in the correct trials (time in correct trials minus time in incorrect trials). The bottom panels show the worst combinations, that were observed most in the incorrect trials (time in incorrect trials minus time in correct trials). The size of the circle reflects the difference in touch duration in correct vs. incorrect trials. Intensity is visualized by color. The y-axis shows the touch locations (the top line in which the whole arm is shaded indicates touches that were applied to at least two individual locations).

To validate if our characterizations of intuitive touch meaningfully reflect the features of touches that are important for communicating emotional cues, we developed standardized touch gestures 468 (Supplementary Movies S1-S6). These were made up of the common features we observed in successful touch communication, and we avoided features found in unsuccessful communication (Figure 3). When delivered by three trained experimenters to naïve strangers in two experiments 
471 (Figure 4), overall identification of the touch messages was significantly better than chance

472 (experiment 3: $E M M F 1$ score $=0.73,95 \% C I=0.62,0.81, z=7.57, p<.001$; experiment 4:

473 EMM F1 score $=0.65,95 \% C I=0.59,0.71, z=19.20, p<.001 ;$ mixed effects models, see Data

474 Analysis for model specification), as was performance for every individual cue (experiment 3: all

$475 p s<.001$, Holm correction; experiment 4: all $p s<=0.02$, Holm correction). As in the intuitive

476 touch task, the correct response was generally the most popular one (except experiment 4

477 gratitude, which was more often labeled calming), with individuals showing more variation

478 (Figure S3). 
A

Experiment 3

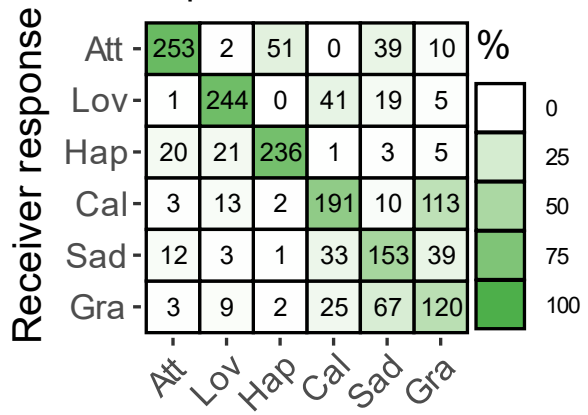

Cued word

C

Experiment 4

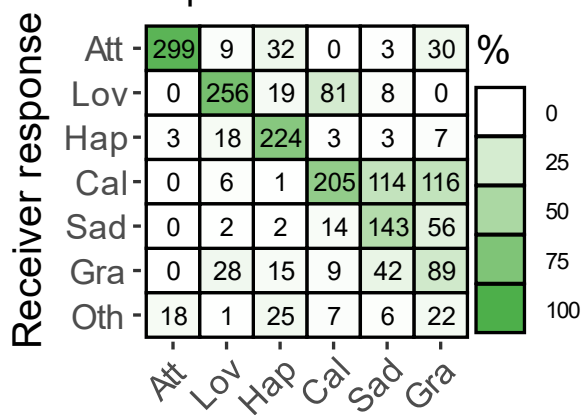

Cued word
B

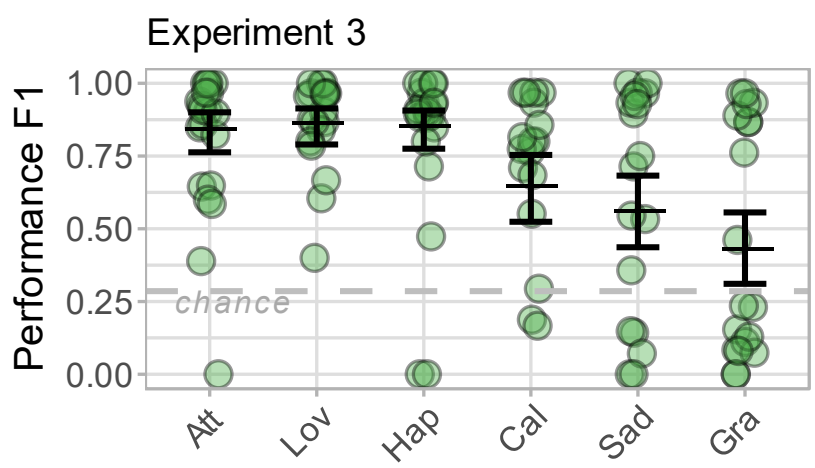

D

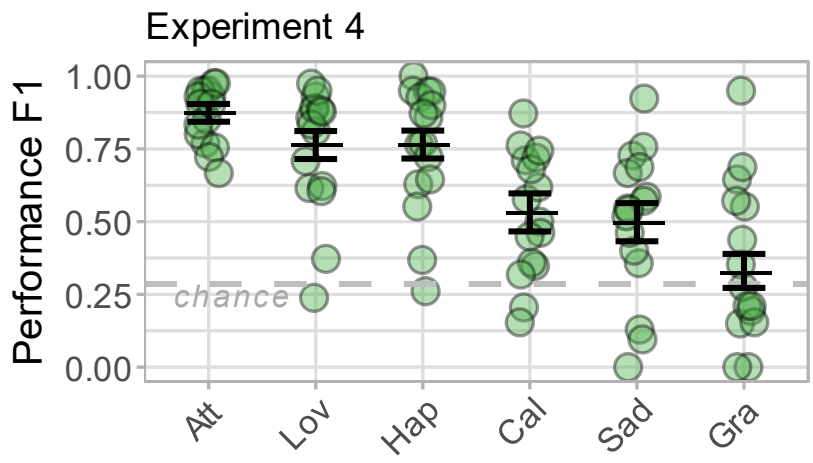

Figure 4. Touch communication between a trained expert sender and a naïve stranger receiver

482 (standardized touch). Performance was better than chance for every cue, demonstrated in two

483 experiments. Cue abbreviations as in Figure 1. (A) Group-level confusion matrix for the communication

484 task involving expert sender 1; experiment 3. Numbers of trials are shown inside the squares, and the

485 shading gives the percentage for that cue. (B) F1 performance score ( $\min =0$, max $=1$ ) for experiment 3.

486 E3 Performance varied significantly by cue $\left(\chi^{2}(5)=1209.80, p<.001\right.$, parametric bootstrap). F1 score

487 for each pair and cue is shown, as well as the model estimate and $95 \%$ confidence interval from the

488 mixed effects model (see text). Chance-level performance $(F 1=0.29)$ is shown as a dashed line. $(\boldsymbol{C})$

489 Group-level confusion matrix from a second experiment with standardized touches (experiment 4), in

490 which participants interpreted the touch messages delivered by both expert senders 2 and 3. (D) F1

491 performance scores, for experiment 4. E4 Performance varied significantly by cue $\left(\chi^{2}(5)=1793.60, p<\right.$

492.001 , parametric bootstrap). F1 score for each pair and cue is shown, as well as the model estimate and

$49395 \%$ confidence interval from the mixed effects model (see text). 
494 Hand tracking using a 3D motion-tracking system (Hauser et al., 2019) was used to characterize

495 the different gestures in more detail, breaking them down into physical primitives (Figure 5A, S4

496 and S5). Both attention and happiness were characterized by high normal velocity, a small

497 contact area and brief duration, but happiness had a high tangential velocity, whereas attention

498 involved almost no lateral movement. Love and calming were both characterized by slow

499 tangential movement; but compared to calming, love had a longer contact duration, a smaller

500 overall contact area, and a higher ratio of finger to palm contact. Sadness was static with a large

501 contact area and long contact duration. Gratitude involved slow normal and tangential

502 movements with moderate contact area and duration.

503 We directly compared the standardized touches performed by experts to the intuitive touches 504 performed by someone in a close relationship in a separate analysis (Figure 5B). We found that 505 identification of the standardized gestures was similar to or even slightly better than the intuitive 506 touches $\left(\chi^{2}(1)=4.52, p=0.027\right.$, parametric bootstrap, mixed effects model, see Data Analysis

507 for full model specification). Specifically, standardized touches produced significantly better 508 recognition than intuitive touches for love, happiness and calming (attention: $O R=0.80,95 \% C I$ $509=0.52,1.22$, z ratio $=-1.04, p=.301$; calming: $O R=1.73,95 \% C I=1.1,2.6, z$ ratio $=2.58, p=$ $510.010 ;$ gratitude: $O R=0.76,95 \% C I=0.50,1.15, z$ ratio $=-1.288, p=.198$; happiness: $O R=$ 5112.32, z ratio $=3.94, p<.001 ;$ love: $O R=4.44,95 \% C I=2.92,6.76, z$ ratio $=6.97, p<0.0001$;

512 sadness: $O R=1.35,95 \% C I=0.89,2.04, z$ ratio $=1.40, p=.161$; post-hoc pairwise

513 comparisons with Holm correction). Furthermore, without feedback, the receivers were able to 514 quickly learn the meaning of the gestures and reached a stable performance level after only 1051512 presentations (Figure 5C). 
A Attention tapping with 1 or 2 fingers

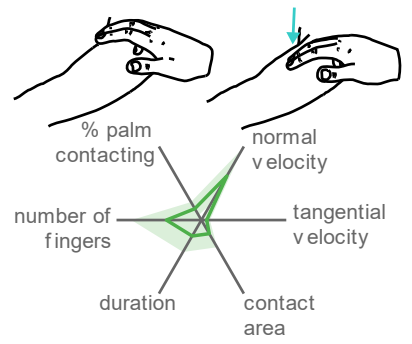

Happiness

tapping with multiple fingers

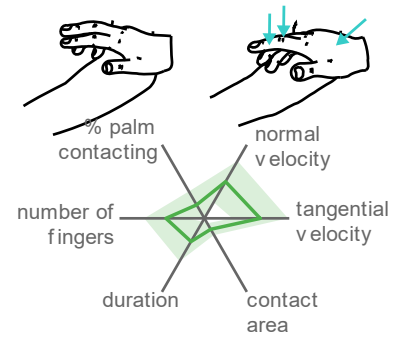

Sadness

gripping with the whole hand

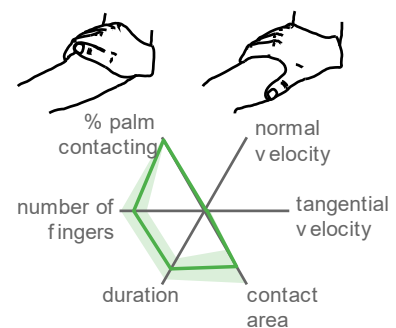

Love

stroking with the fingers

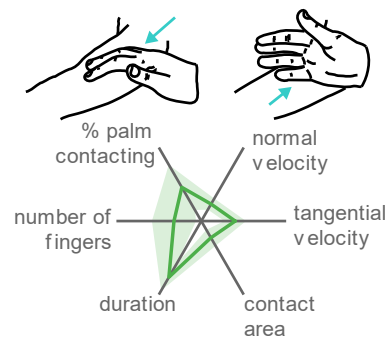

Calming

stroking with the whole hand

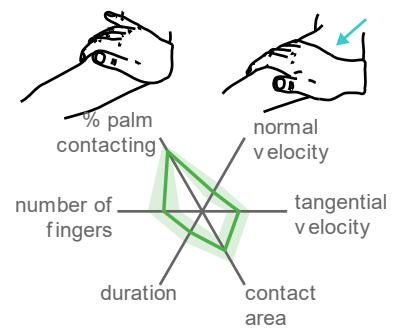

Gratitude

patting and stroking with the whole hand

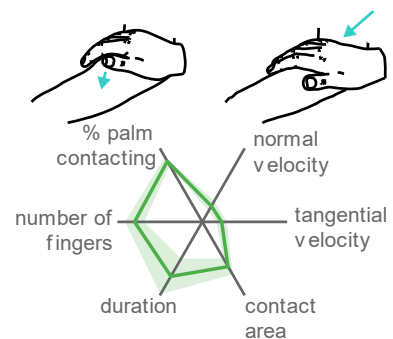

B

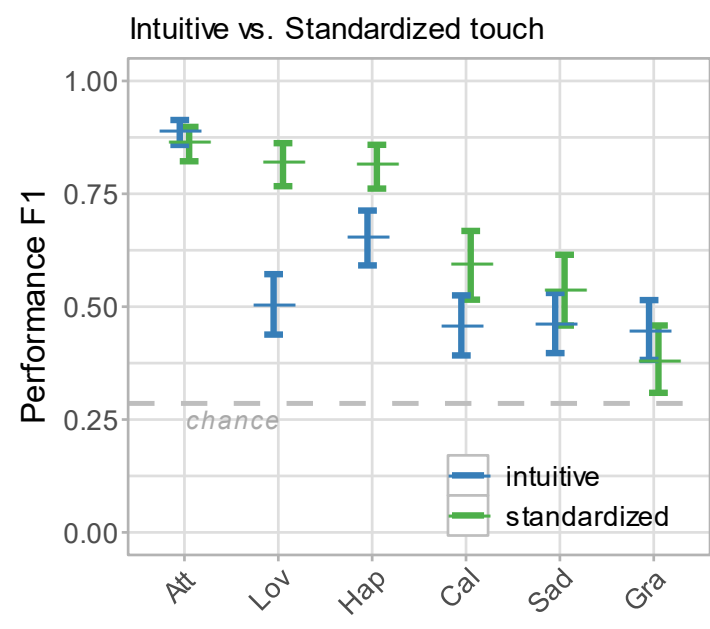

C

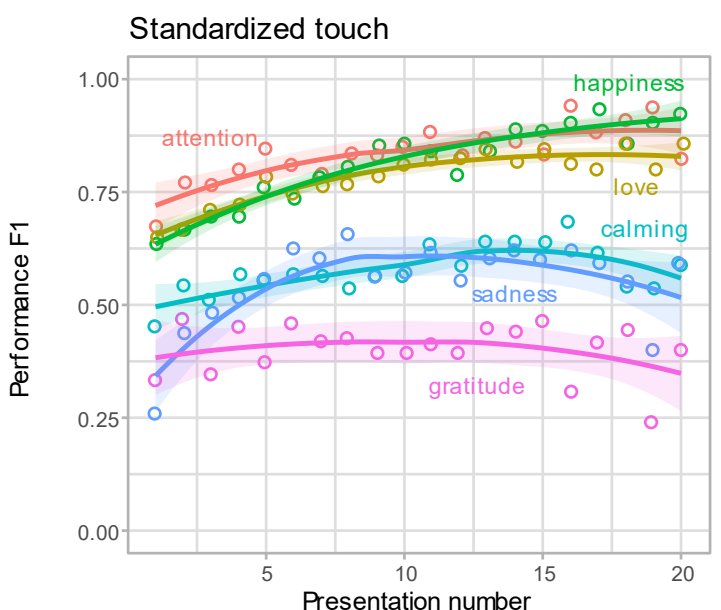

Figure 5. Characterization and evaluation of the standardized touches. Abbreviations as in Figure 1. (A)

Physical features of the standardized touches delivered by trained experimenters and measured using an automated hand-tracking system (experiment 4, experts 2 and 3). Mean values for each contact metric were normalized between 0 and 1 based on the population standard deviation. (B) Model estimates of F1 performance score, and 95\% CIs from a mixed-effects model comparing intuitive and standardized touch strategies. Performance varied significantly by cue $\left(\chi^{2}(5)=4452.44, p=0.001\right.$, parametric bootstrap),

523 and there was a significant interaction between touch type and cue $\left(\chi^{2}(5)=697.12, p=0.001\right.$,

524 parametric bootstrap). (C) The standardized cues were learned quickly, with performance reaching a 525 plateau within 10-12 presentations for most cues, without feedback. The lines were fit with a loess 526 function with a span of 1, the shaded area is the 95\% CI based on the standard error (combined data 527 from experiments 3 and 4).

\section{Discussion}


529 We have identified touch expressions for which the social and emotional content are broadly

530 understood between strangers, at least within one cultural context. Indeed, skillful strangers can

531 communicate emotional touch messages as effectively or better than people in close

532 relationships. We have ruled out a large role for an idiosyncratic touch language developed

533 within couples or other close relationships in touch communication efficacy. Communication

534 performance without feedback was well above chance with our standardized touch expressions

535 reflecting their basis in real interpersonal touch interactions, and a common understanding.

536 However, our study recruited adults living in Sweden, and an important open question is whether

537 the standardized touch expressions are universally understood across cultural context.

538 That people can understand social and emotional touch messages confirms earlier reports (App et

539 al., 2011; Hertenstein et al., 2009; Hertenstein, Keltner, et al., 2006; Kirsch et al., 2018). That

540 strangers are as good or better at communicating via touch than people in a close relationship

541 provides an apparent contrast with earlier work showing that romantic couples communicate

542 more effectively than stranger pairs (Thompson \& Hampton, 2011). We reconcile this by

543 considering that the earlier work used untrained strangers, while we trained our experimenters to

544 use effective touch strategies using clear and distinct touch gestures. This training may

545 compensate for a greater reluctance to touch a stranger compared to someone close (Suvilehto et

546 al., 2015, 2019).

547 We went beyond previous studies' (Gaus et al., 2015; Hertenstein et al., 2009; Hertenstein,

548 Keltner, et al., 2006; Jung et al., 2015; Masson \& Op de Beeck, 2018; Supratman et al., 2020)

549 efforts to characterize the physical features of touch communication. We did this by first

550 evaluating their contribution to communication efficacy, and then validating this by showing that

551 standardized touch strategies based on the features identified as effective, were indeed capable of 
552 conveying the intended message. Additionally, we used a 3D hand-tracking approach to obtain

553 quantitative descriptors of the standardized touch expressions including normal and tangential

554 velocity, contact area and duration, and palm and finger contact.

555 A previous study evaluated the relationship between motion energy obtained from filmed

556 interpersonal touch interactions and observers' judgments of their valence and arousal (Masson

$557 \&$ Op de Beeck, 2018). More positively viewed interactions tend to have lower motion energy,

558 while more (emotionally) arousing interactions have higher motion energy. Our results are

559 consistent with this finding, with the highest measured velocities observed in our attention and

560 happiness expressions, which are likely to be relatively high arousal. Interestingly, these are the

561 same two cues that in the receiver provoked a significant increase in zygomatic activity, which

562 has been associated with high arousal positive stimuli (Fujimura et al., 2010). Additionally, our

563 sadness expression, being the only negative valence message, had near zero velocity.

564 While measuring motion energy is quantitative and informative, we have shown that more

565 detailed physical measurements can further discriminate specific touch messages, e.g., happiness

566 and attention had similar normal velocities but very different tangential velocities, while contact

567 duration and area discriminated love and calming, which had similar tangential velocities. This is

568 consistent with studies of human-delivered touch to pressure sensor surfaces (Gaus et al., 2015;

569 Supratman et al., 2020) and robot-delivered touch to humans (Teyssier et al., 2020) showing that

570 a variety of contact area and motion dynamics measures are required to distinguish different

571 touch actions.

572 In our touch communication task, the sender and receiver could not see each other's expressions,

573 but we asked if either role's facial expressions might interact with touch communication. The

574 senders' zygomatic and corrugator activity, as well as the receivers' zygomatic activity varied 
575 according to the touch message. However, when we looked for a relationship between facial

576 muscle activity and communication success on an individual level, we did not find it. This

577 suggests that the varying facial muscle activity may simply reflect the participants' adoption of

578 an existing set of strategies for communicating emotion that would normally involve both touch

579 and facial expression. An alternative explanation for these findings is that some aspects of facial

580 muscle activity that we did not measure are relevant to touch communication, or that there is a

581 weak relationship (small effect size) that we failed to detect.

582 The senders showed increased zygomatic activity when communicating attention, happiness and

583 gratitude, suggesting that expressing these messages may be accompanied by smiling. The

584 sender also showed increased corrugator activity when communicating happiness, possibly due

585 to high arousal produced by its high intensity (Figure 3) and speed (Figure 5). The receivers also

586 showed increased zygomatic activity when feeling the touch expressions for attention and

587 happiness, but not gratitude. The relatively high intensity and speed of the attention and

588 happiness may have provoked a positive emotional response from the receiver (Fujimura et al.,

589 2010). The result contrasts with previously observed increased zygomatic activity in response to

590 a gentle stroking with a brush that most resembled our calming expression (Pawling et al., 2017).

591 The touch evoked no significant changes in the corrugator activity of the receivers, in contrast to

592 previous studies showing reduced corrugator activity (Mayo et al., 2018; Ree et al., 2020) in

593 response to pleasant touch.

594 Our standardized touches closely resemble authentic interpersonal touch, while being optimized

595 for communicating specific messages. This is because they were developed based on the

596 successful communication strategies that people in close relationships used when given minimal

597 instructions. While we cued a specific message to communicate, and limited the area of the body 
598 that could be touched, the participants were otherwise free to choose their own touch strategy.

599 Some previous studies on touch communication systematically investigated different types of

600 messages. For example, App et al. (2011) investigated which non-verbal communication

601 channels are used for messages that correspond to different social functions (status-conveying,

602 survival-focused and intimacy-focused). Kirsch et al. (2018) investigated how the same touches

603 were interpreted differently if the receivers were given different tasks - either interpreting the

604 emotion or the intention of the person performing the touches. In our study, we did not try to

605 systematically vary details of the messages to be communicated because we did not have any

606 specific hypotheses relating to them. Rather, our goal was simply to capture a variety of touch

607 behaviors. Similarly, we did not did not control the exact type of relationship or demographics of

608 our participants (Hertenstein et al., 2009; Hertenstein, Keltner, et al., 2006; Thompson \&

609 Hampton, 2011), the investigation of which would have required different sample size

610 considerations.

611 The interpretation of touch messages is likely to be influenced by a large number of contextual

612 factors (Cekaite \& Bergnehr, 2018; Weiss, 1986). We found that when pairs swapped roles,

613 performance improved (Figure 1B-D). Experiencing multiple roles may have aided

614 communication in a way that is similar to more interactive, natural contexts. One example of a

615 real-world situation that has similar contextual information to our communication task is text

616 communication. In principle, text alone can convey all information, analogous to speech.

617 However, the widespread use of emoji to convey facial expressions illustrates that there is a clear

618 desire to use analogs of non-verbal communication. Emoji rely on a pre-existing broad consensus

619 about the mapping between the visual elements of a facial expression and an emotional meaning.

620 Similarly, our standardized touches did not require specific training or any feedback, but were 
621 able to capitalize on a pre-existing broad understanding, while also benefiting from additional

622 contextual information.

\section{Conclusions}

624 When people in a close relationship engaged in emotional touch communication, the senders

625 intuitively produced distinct gestures to effectively communicate different messages of attention,

626 love, happiness, calming, sadness, and gratitude. The touch messages were associated with the

627 senders' facial expressions, but we found no evidence that this was related to the ability of the

628 receivers to correctly interpret the touch message. In contrast, choosing the right touch strategies

629 with appropriate physical features were sufficient for effective communication. We demonstrated

630 this with our set of tactile standardized gestures delivered to naïve strangers, which were

631 identified even more successfully than the intuitive gestures delivered within the context of a

632 close relationship. We have thus presented a lexicon of gestures, with distinct physical

633 characteristics, that can be used for social communication to enrich emotional content. These

634 findings inform the development and improvement of interpersonal communication mediated by

635 haptic interfaces. It could also inform development of training for people in any context in which

636 it is desirable that communication is enhanced and emotional touch communication is

637 appropriate. It may also provide a useful tool for exploring social differences associated with 638 psychiatric disorders. 
641 This study was funded by Facebook Inc and the Swedish Research Council. The experiments

642 were initiated and designed by researchers at Linköping University in Sweden, and University of

643 Virginia and Columbia University in the United States. All experiments were carried out at

644 Linköping University, Sweden. Facebook influenced the broad topic of the research, and

645 encouraged publication without influencing the interpretation of the results. The co-authors

646 employed by Facebook contributed scientifically, and were not under any specific directive

647 about the reporting or interpretation of the results.

\section{Author contributions}

649 Conceptualization SM, RB, GN, SSN, AI, EAL, FA, GJG, HO; Methodology SM, SCH, RB, 650 AM, PMI, GN, GJG, HO; Software SM, SCH, AK, GJG; Investigation SM, AK, RB, AM, PMI, 651 LH; Formal Analysis SM, SCH, AK; Writing - Original draft SM, RB, AM, HO; Writing 652 Review \& Editing SM, SCH, AK, RB, AM, PMI, LH, GN, SSN, AI, EAL, FA, GJG, HO

\section{Acknowledgements}

654 We wish to thank Leah Mayo and Anna Asratian who advised us on facial EMG methods, Juulia 655 Suvilehto who suggested using F1 score, an anonymous reviewer who suggested improvements 656 to the analysis of the facial EMG data, and India Morrison and Marcus Heilig who read and gave 657 feedback on an early version of the manuscript. 
660 App, B., McIntosh, D. N., Reed, C. L., \& Hertenstein, M. J. (2011). Nonverbal channel use in communication of emotion: How may depend on why. Emotion, 11(3), 603-617. https://doi.org/10.1037/a0023164

663 Cekaite, A., \& Bergnehr, D. (2018). Affectionate touch and care: Embodied intimacy, compassion and control in early childhood education. European Early Childhood

Cekaite, A., \& Kvist Holm, M. (2017). The Comforting Touch: Tactile Intimacy and Talk in Managing Children's Distress. Research on Language and Social Interaction, 50(2), 109-127. https://doi.org/10.1080/08351813.2017.1301293 Education Research Journal, 26(6), 940-955. https://doi.org/10.1080/1350293X.2018.1533710

Dunbar, R. I. M. (2010). The social role of touch in humans and primates: Behavioural function and neurobiological mechanisms. Neuroscience \& Biobehavioral Reviews, 34(2), 260268. https://doi.org/10.1016/j.neubiorev.2008.07.001

Fridlund, A. J., \& Cacioppo, J. T. (1986). Guidelines for human electromyographic research. Psychophysiology, 23(5), 567-589. https://doi.org/10.1111/j.1469-8986.1986.tb00676.x mimicry. International Journal of Psychophysiology, 76(2), 88-92. https://doi.org/10.1016/j.ijpsycho.2010.02.008 
681 Gaus, Y. F. A., Olugbade, T., Jan, A., Qin, R., Liu, J., Zhang, F., Meng, H., \& Bianchi-

682 Berthouze, N. (2015). Social Touch Gesture Recognition using Random Forest and

683 Boosting on Distinct Feature Sets. Proceedings of the 2015 ACM on International

684 Conference on Multimodal Interaction - ICMI '15, 399-406.

685 https://doi.org/10.1145/2818346.2830599

686 Hauser, S. C., McIntyre, S., Israr, A., Olausson, H., \& Gerling, G. J. (2019). Uncovering Humanto-Human Physical Interactions that Underlie Emotional and Affective Touch Communication. 2019 IEEE World Haptics Conference (WHC), 407-412. https://doi.org/10.1109/WHC.2019.8816169

Hertenstein, M. J. (2002). Touch: Its Communicative Functions in Infancy. Human Development, 45(2), 70-94. https://doi.org/10.1159/000048154

Hertenstein, M. J., Holmes, R., McCullough, M., \& Keltner, D. (2009). The communication of emotion via touch. Emotion, 9(4), 566-573. https://doi.org/10.1037/a0016108

Hertenstein, M. J., Keltner, D., App, B., Bulleit, B. A., \& Jaskolka, A. R. (2006). Touch communicates distinct emotions. Emotion, 6(3), 528-533. https://doi.org/10.1037/15283542.6.3.528

Hertenstein, M. J., Verkamp, J. M., Kerestes, A. M., \& Holmes, R. M. (2006). The Communicative Functions of Touch in Humans, Nonhuman Primates, and Rats: A Review and Synthesis of the Empirical Research. Genetic, Social, and General

701 Jerritta, S., Murugappan, M., Wan, K., \& Yaacob, S. (2014). Emotion recognition from facial 702 EMG signals using higher order statistics and principal component analysis. Journal of 
the Chinese Institute of Engineers, 37(3), 385-394. https://doi.org/10.1080/02533839.2013.799946

Jung, M. M., Cang, X. L., Poel, M., \& MacLean, K. E. (2015). Touch Challenge '15: Recognizing Social Touch Gestures. Proceedings of the 2015 ACM on International Conference on Multimodal Interaction - ICMI'15, 387-390. https://doi.org/10.1145/2818346.2829993

709

Kirsch, L. P., Krahé, C., Blom, N., Crucianelli, L., Moro, V., Jenkinson, P. M., \& Fotopoulou, A. (2018). Reading the mind in the touch: Neurophysiological specificity in the communication of emotions by touch. Neuropsychologia, 116, 136-149. https://doi.org/10.1016/j.neuropsychologia.2017.05.024

713 Künecke, J., Hildebrandt, A., Recio, G., Sommer, W., \& Wilhelm, O. (2014). Facial EMG 714 Responses to Emotional Expressions Are Related to Emotion Perception Ability. PLoS 715

Larsen, J. T., Norris, C. J., \& Cacioppo, J. T. (2003). Effects of positive and negative affect on electromyographic activity over zygomaticus major and corrugator supercilii. Psychophysiology, 40(5), 776-785. https://doi.org/10.1111/1469-8986.00078

Masson, H. L., \& Op de Beeck, H. (2018). Socio-affective touch expression database. PLOS ONE, 13(1), e0190921. https://doi.org/10.1371/journal.pone.0190921

721 Mayo, L. M., Lindé, J., Olausson, H., Heilig, M., \& Morrison, I. (2018). Putting a good face on 722 723 touch: Facial expression reflects the affective valence of caress-like touch across modalities. Biological Psychology, 137, 83-90. https://doi.org/10.1016/j.biopsycho.2018.07.001 
McIntyre, S., Moungou, A., Boehme, R., Isager, P. M., Lau, F., Israr, A., Lumpkin, E. A., Abnousi, F., \& Olausson, H. (2019). Affective touch communication in close adult relationships. 2019 IEEE World Haptics Conference (WHC), 175-180. https://doi.org/10.1109/WHC.2019.8816093

Murphy, K., \& Garavan, H. (2004). An empirical investigation into the number of subjects required for an event-related fMRI study. NeuroImage, 22(2), 879-885. https://doi.org/10.1016/j.neuroimage.2004.02.005

Pawling, R., Cannon, P. R., McGlone, F. P., \& Walker, S. C. (2017). C-tactile afferent stimulating touch carries a positive affective value. PLOS ONE, 12(3), e0173457. https://doi.org/10.1371/journal.pone.0173457

Pedersen, T. L. (2017). patchwork: The Composer of ggplots ( $\mathrm{R}$ package version 0.0.1) [Computer software]. https://github.com/thomasp85/patchwork

Pedregosa, F., Varoquaux, G., Gramfort, A., Michel, V., Thirion, B., Grisel, O., Blondel, M., Prettenhofer, P., Weiss, R., Dubourg, V., Vanderplas, J., Passos, A., Cournapeau, D., Brucher, M., Perrot, M., \& Duchesnay, É. (2011). Scikit-learn: Machine Learning in Python. Journal of Machine Learning Research, 12(85), 2825-2830.

Peirce, J. W. (2007). PsychoPy_Psychophysics software in Python. Journal of Neuroscience Methods, 162(1-2), 8-13. https://doi.org/10.1016/j.jneumeth.2006.11.017

Picard, R. W., Vyzas, E., \& Healey, J. (2001). Toward machine emotional intelligence: Analysis of affective physiological state. IEEE Transactions on Pattern Analysis \& Machine Intelligence, 23, 1175-1191. https://doi.org/10.1109/34.954607

R Core Team. (2018). R: A language and environment for statistical computing. R Foundation for Statistical Computing. https://www.R-project.org/ 
Ree, A., Bendas, J., Pabel, L., Croy, I., \& Sailer, U. (2020). Right between the eyes: Corrugator muscle activity tracks the changing pleasantness of repeated slow stroking touch. Physiology \& Behavior, 222, 112903. https://doi.org/10.1016/j.physbeh.2020.112903

Singmann, H., \& Kellen, D. (2019). An Introduction to Mixed Models for Experimental Psychology. In D. Spieler \& E. Schumacher (Eds.), New Methods in Cognitive Psychology (1st ed., pp. 4-31). Routledge. https://doi.org/10.4324/9780429318405-2

Supratman, J. C., Hayashibara, Y., \& Irie, K. (2020). Recognizing Human Emotion Based on

Suvilehto, J. T., Glerean, E., Dunbar, R. I. M., Hari, R., \& Nummenmaa, L. (2015). Topography Applied Force. 2020 13th International Conference on Human System Interaction (HSI), emotions through touch. Cognition \& Emotion, 25(2), 295-306.

https://doi.org/10.1080/02699931.2010.492957 
771 Tin Kam Ho. (1995). Random decision forests. Proceedings of 3rd International Conference on

772 Document Analysis and Recognition, 1, 278-282.

$773 \quad$ https://doi.org/10.1109/ICDAR.1995.598994

774 Tsalamlal, M. Y., Amorim, M.-A., Martin, J.-C., \& Ammi, M. (2018). Combining Facial

775 Expression and Touch for Perceiving Emotional Valence. IEEE Transactions on Affective

776 Computing, 9(4), 437-449. https://doi.org/10.1109/TAFFC.2016.2631469

777 Tsalamlal, M. Y., Martin, J.-C., Ammi, M., Tapus, A., \& Amorim, M.-A. (2015). Affective

778 handshake with a humanoid robot: How do participants perceive and combine its facial

779 and haptic expressions? 2015 International Conference on Affective Computing and

780 Intelligent Interaction (ACII), 334-340. https://doi.org/10.1109/ACII.2015.7344592

781 Weiss, S. J. (1986). Psychophysiologic effects of caregiver touch on incidence of cardiac

782 dysrhythmia. Heart \& Lung: The Journal of Critical Care, 15(5), 495-505.

783 Wickham, H. (2016). ggplot2: Elegant Graphics for Data Analysis. Springer-Verlag New York.

$784 \quad$ https://ggplot2.tidyverse.org

785

786 
787 The language of social touch is intuitive and quantifiable

788 Supplementary Text

789 Questionnaires

790 In experiments $1-3$, participants answered questionnaires at the beginning of the experimental

791 session. Data from experiment 2 are reported in a separate manuscript, and data from

792 experiments 1 and 3 are given here. Results from all three experiments were similar. Participants

793 in both experiments 1 and 3 answered the 'Social Touch Questionnaire' (STQ) a 20-item

794 validated questionnaire that assesses the personal attitude towards social situations involving

795 touch (Wilhelm et al., 2001). In experiment 1, participants were also asked to define the nature of

796 their relationship, which was categorized as romantic, friends or family. Participants were also

797 asked to rate the degree of emotional closeness.

798 Social Touch Questionnaire

799 The Social Touch Questionnaire (STQ) has a minimum score of 0, indicating relative comfort

800 with touch, and a maximum of 80 , indicating relative discomfort with touch. The overall mean

801 score in experiment 1 was $29.0(\mathrm{SD}=9.6)$. STQ scores did not significantly differ between our

802 female $(\mathrm{M}=31.2, \mathrm{SD}=9.3)$ and male $(\mathrm{M}=27.1, \mathrm{SD}=9.7)$ participants $(95 \% \mathrm{CI}=-2.8,11.1$,

$803 \mathrm{t}(30)=1.2, \mathrm{p}=0.231)$. There was no significant correlation between touch communication

804 performance and STQ scores of the receiver $(r=-0.02, \mathrm{CI}=-0.38,0.32, \mathrm{p}=0.923)$, or of the

805 sender $(r=0.12, C I=-0.23,0.46, p=0.511)$. Similarly, in experiment 3 , performance on the 
806 touch communication task did not significantly correlate with STQ scores (receiver: $\mathrm{r}=0.06, \mathrm{p}=$ 807 0.799).

\section{Relationship closeness}

809 In Experiment 1, participants rated the relationship with their partner for closeness, on a scale of

$8101-4$. Because of the small range in scores, we treated closeness as a categorical variable.

811 Overall touch communication performance was not significantly affected by the closeness score

812 provided by the participants (receiver: $\mathrm{F}(2,28)=0.7, \mathrm{p}=0.493$, CI: \pm 0.5 ; sender: $\mathrm{F}(2,28)=1.3, \mathrm{p}$ $813=0.299, \mathrm{CI}: \pm 0.5)$.

\section{References}

815 Wilhelm, F. H., Kochar, A. S., Roth, W. T., \& Gross, J. J. (2001). Social anxiety and response to 816 touch: Incongruence between self-evaluative and physiological reactions. Biological

817 Psychology, 58(3), 181-202. https://doi.org/10.1016/S0301-0511(01)00113-2 


\section{Supplementary Figures}
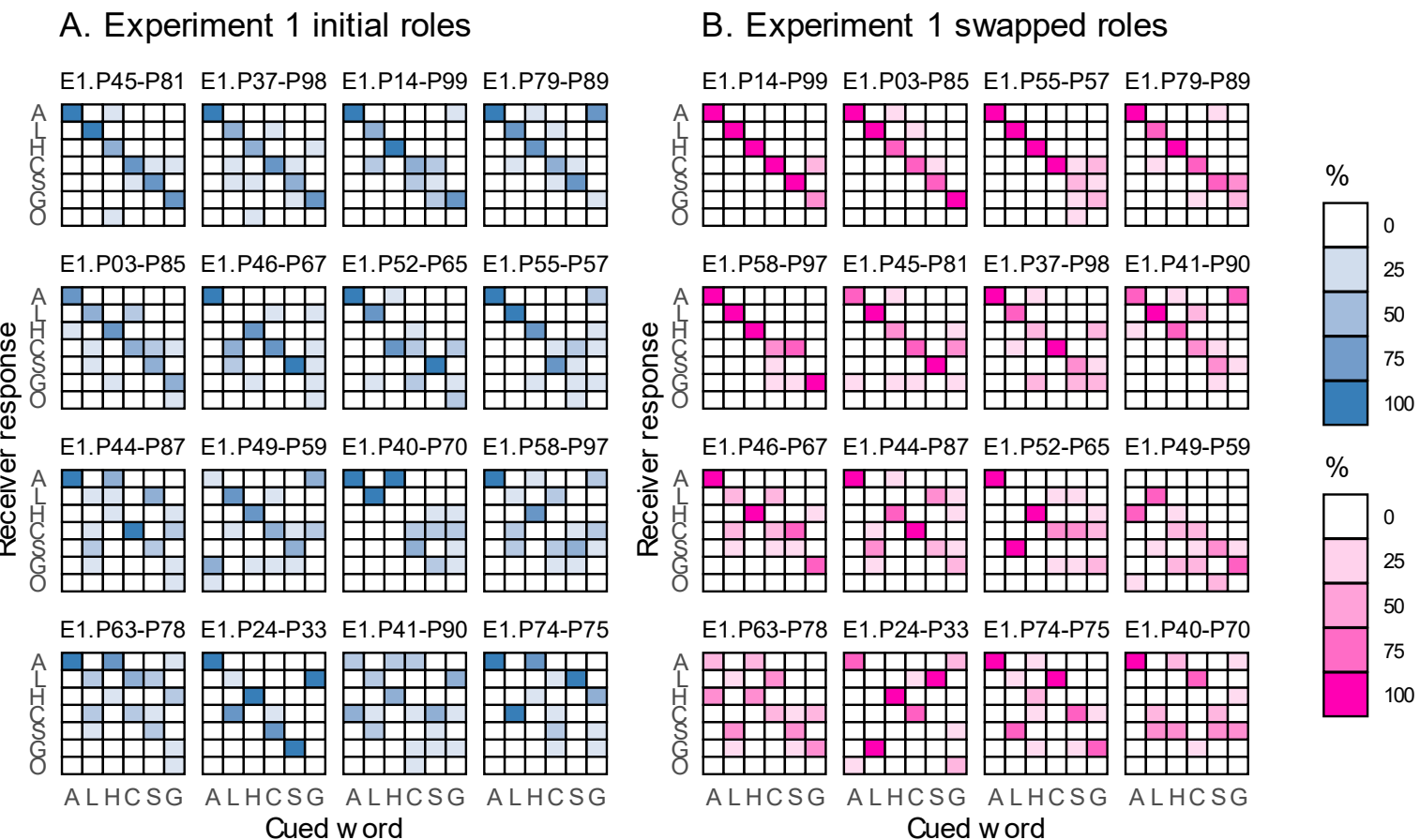

\section{Experiment 2}
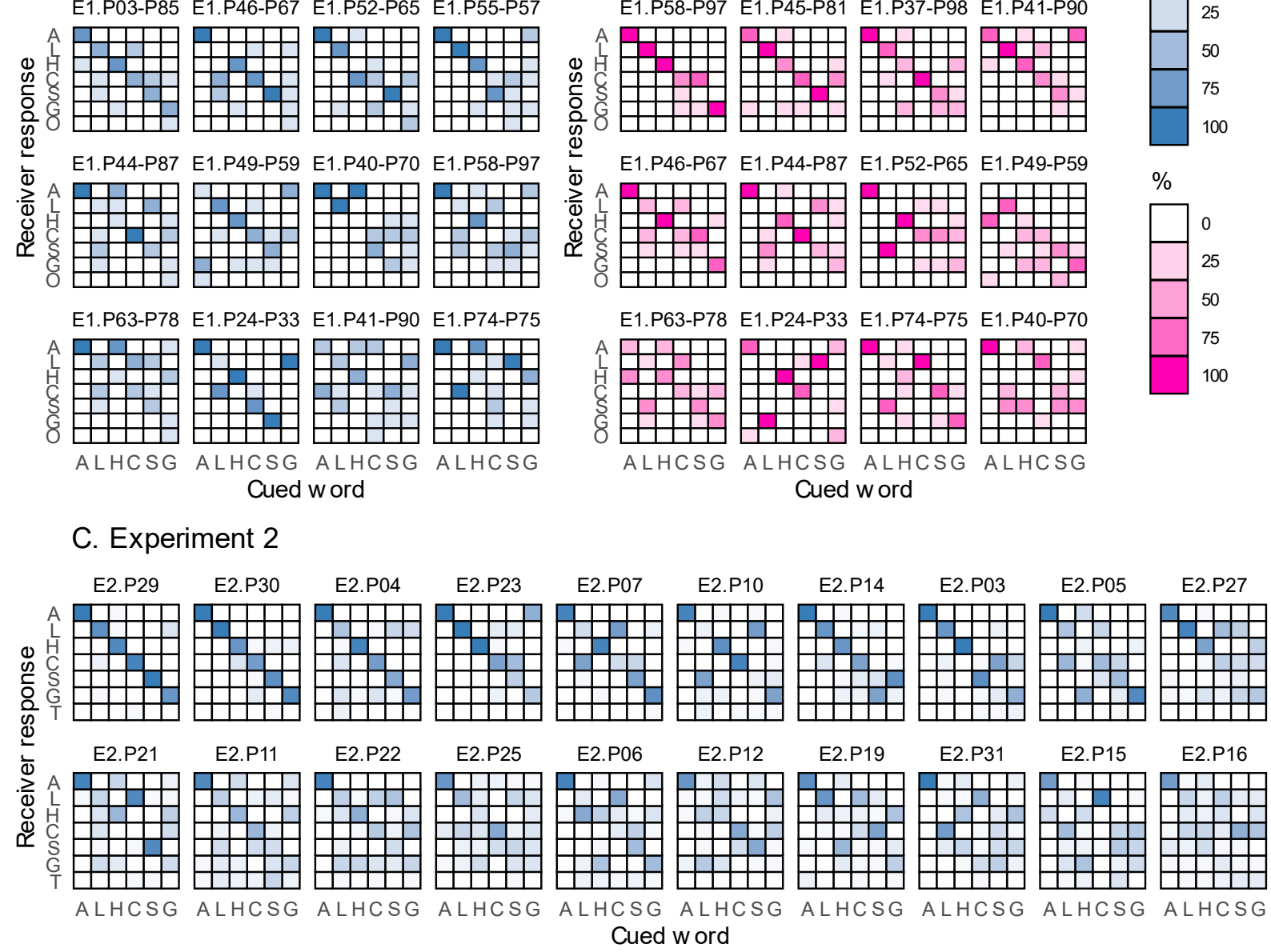

Cued word

Cued w ord

822 Figure S1. Individual confusion matrices for the intuitive touch communication task between people in a

823 close personal relationship. Each panel shows the confusion matrix for one participant in the receiver

824 role. Panels are arranged in order of overall performance. $A=$ attention, $L=$ love, $H=$ happiness, $C=$

825 calming, $S=$ sadness, $G=$ gratitude, $O=$ other, $T=$ timeout. 
$\rightarrow$ attention

A. Sender Zygomaticus

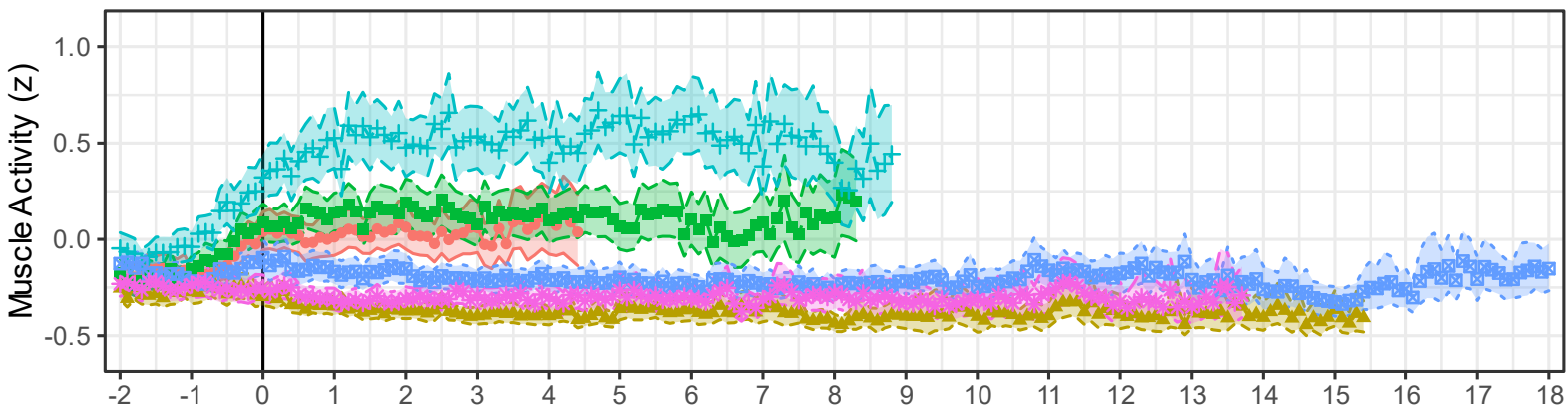

B. Receiver Zygomaticus

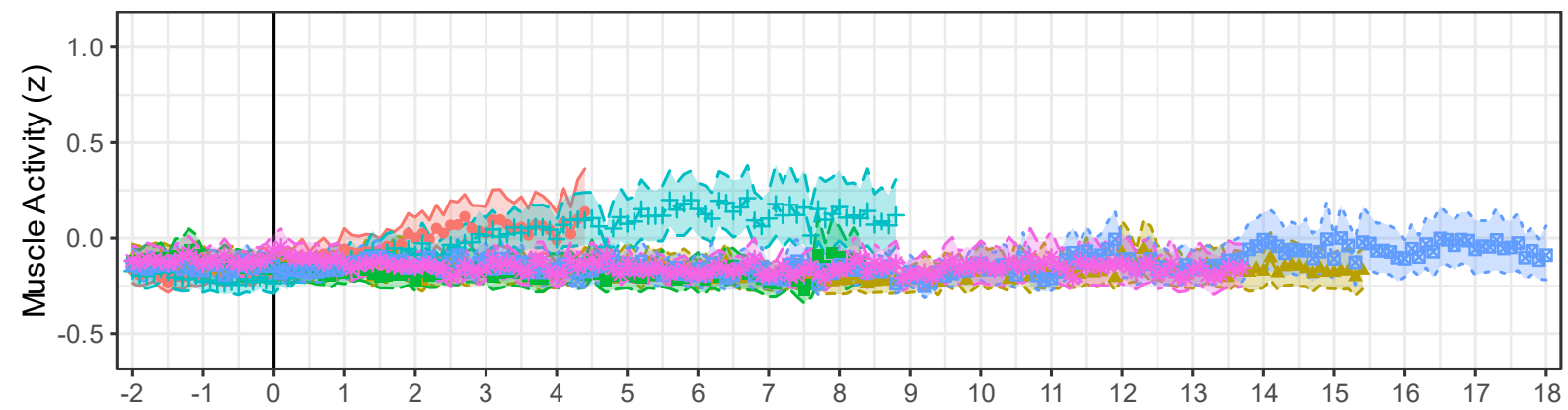

C. Sender Corrugator

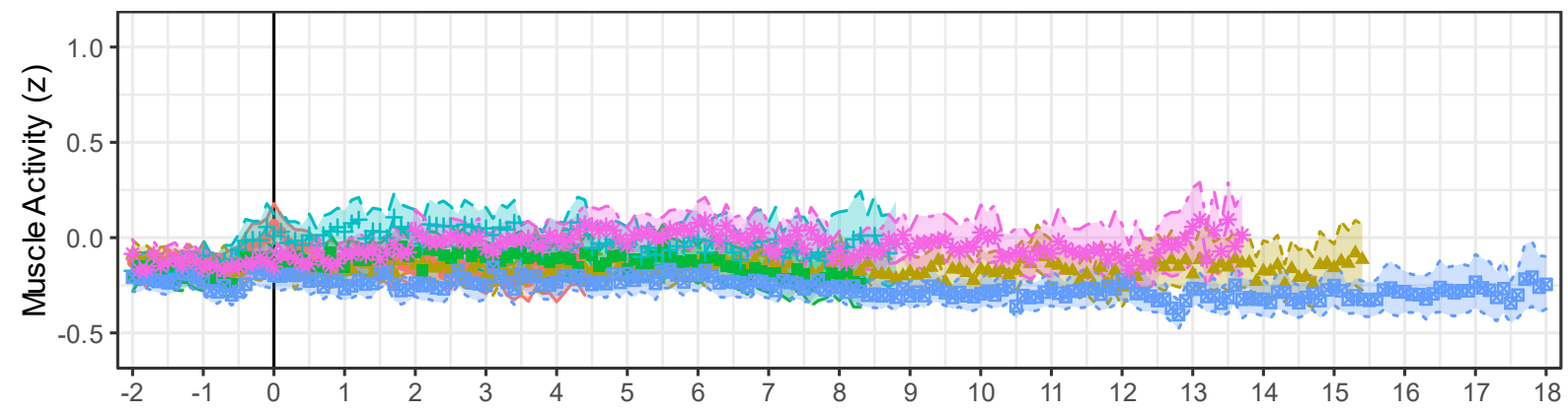

D. Receiver Corrugator

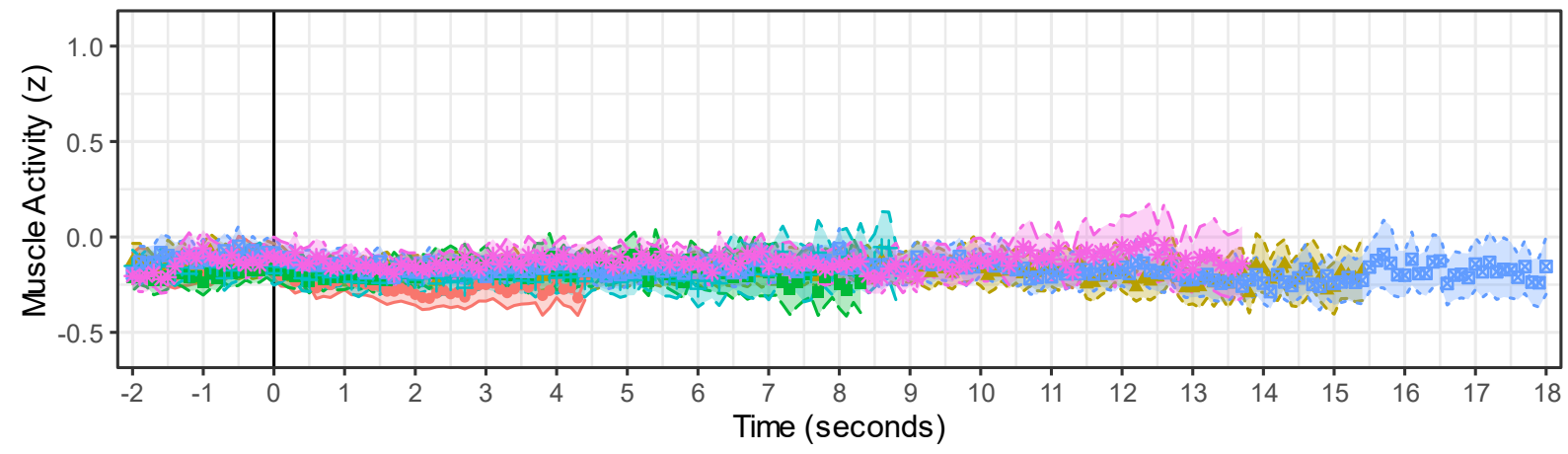


827 Figure S2. Facial EMG over time during the touch communication task. Data are in 100ms bins, shaded 828 area $=95 \%$ CI based on 1000 bootstrapped samples, mid-points are the mean. 0 sec $=$ touch onset. Data 829 for each cued word are plotted until the time point at which $75 \%$ of trials have finished.

A. Experiment 3

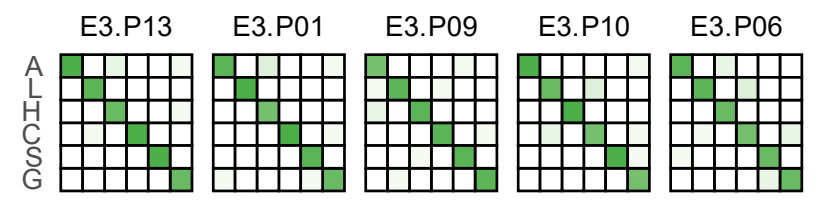

\begin{abstract}
E3.P03
\end{abstract}
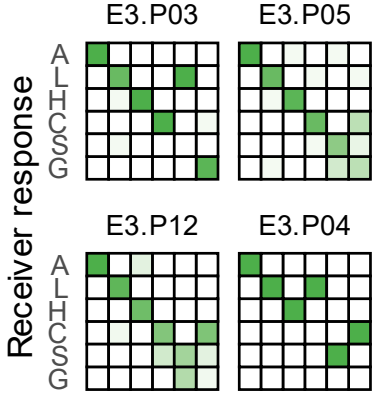

E3.P15

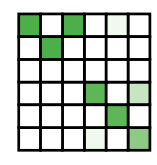

E3.P16

E3.P17
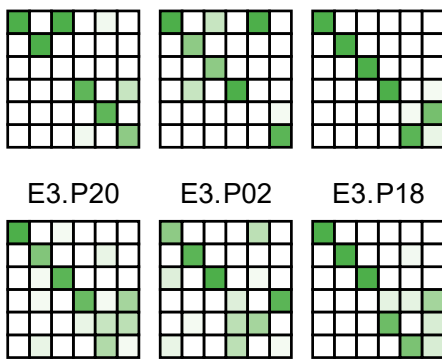

E3.P18
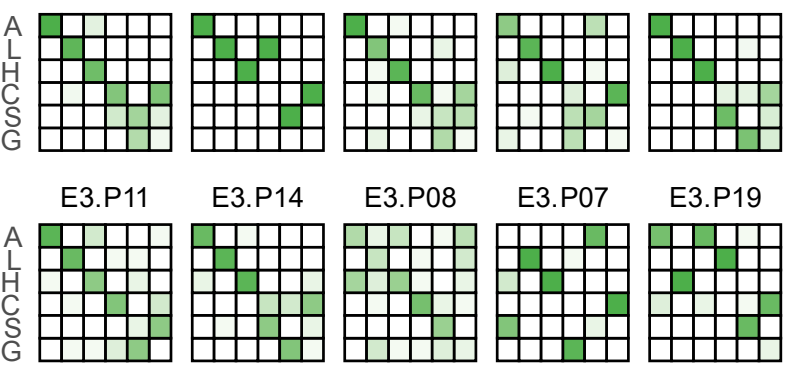

ALHCSG ALHCSG
B. Experiment 4
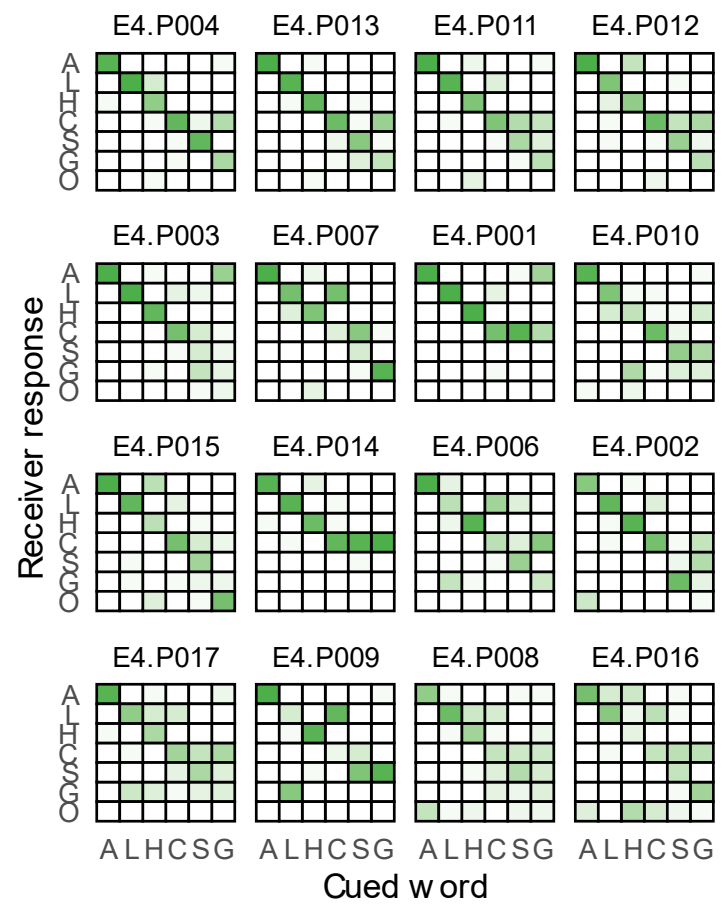

830

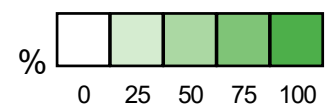

831 Figure S3. Individual confusion matrices for the expert touch communication task where the sender was a

832 trained experimenter unknown to the receiver. Each panel shows the confusion matrix for one participant

833 in the receiver role. Panels are arranged in order of overall performance. $A=$ attention, $L=$ love, $H=$

834 happiness, $C=$ calming, $S=$ sadness, $G=$ gratitude, $O=$ other. 


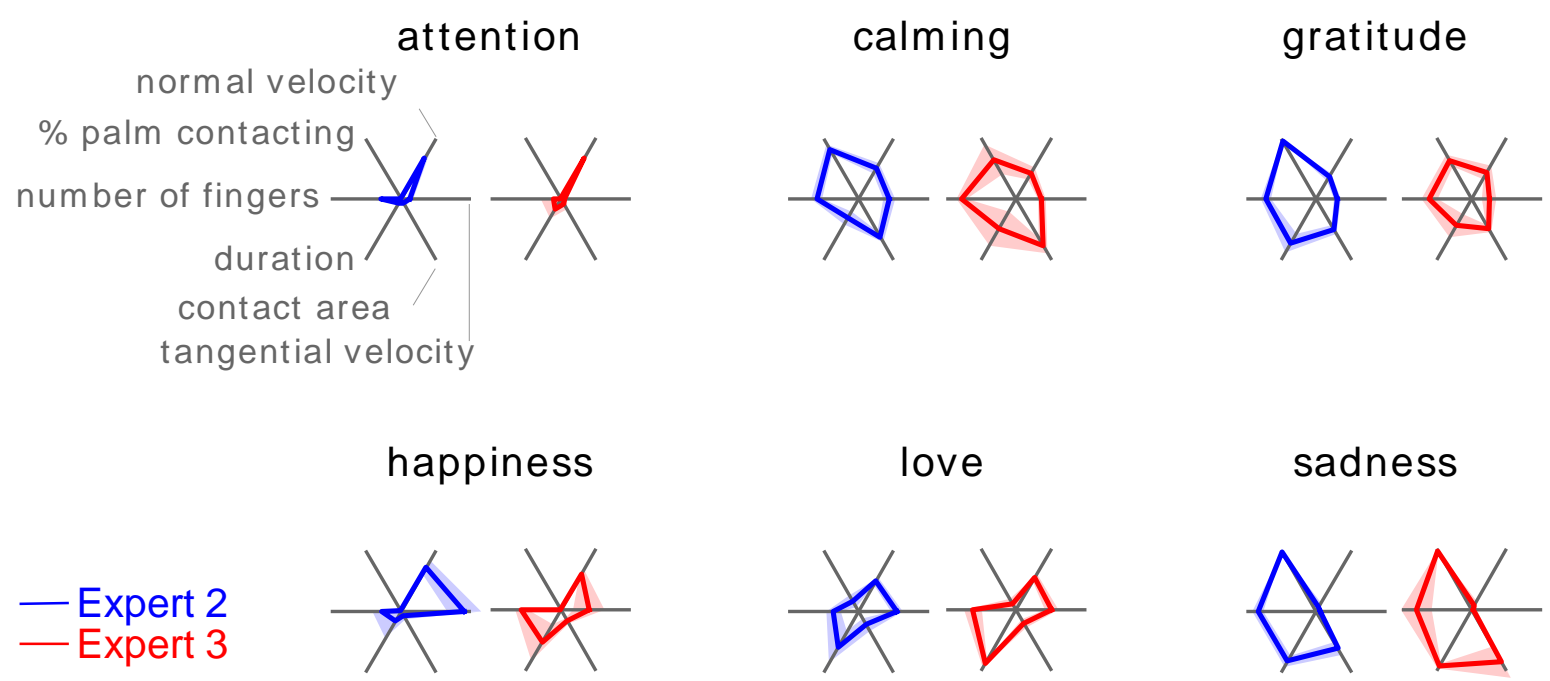

836 Figure S4. Separate representation of the physical features of the standardized touches for two trained

837 experimenters, measured using an automated hand-tracking system (experiment 4). Mean values for each 838 contact metric were normalized between 0 and 1 based on the population standard deviation. 
calming
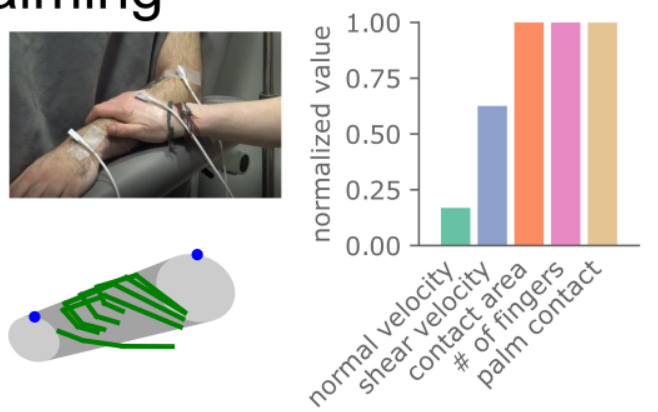

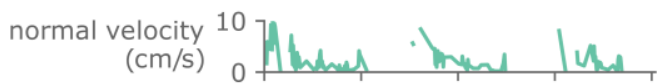

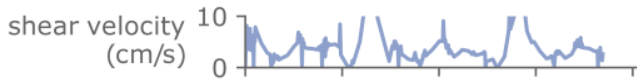
contact area $50-1$
$\left(\mathrm{~cm}^{2}\right)$

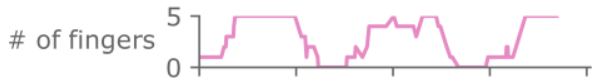

contact

palm contact

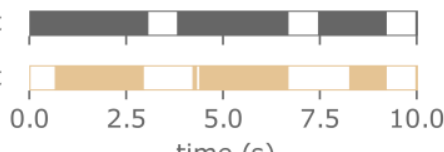

\section{sadness}

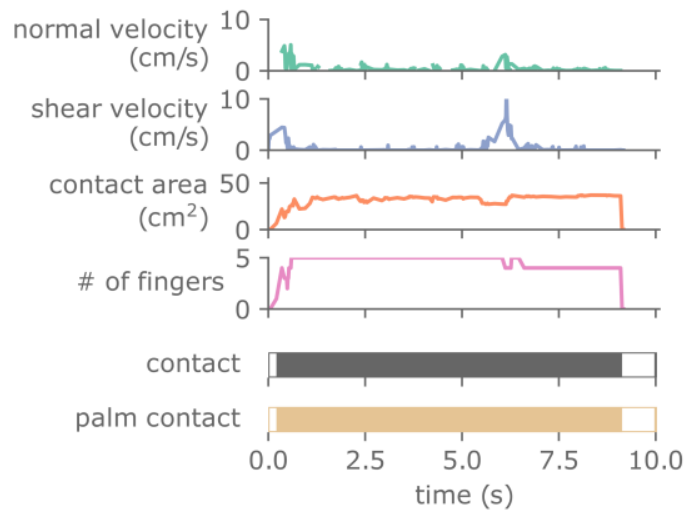

\section{love}

attention
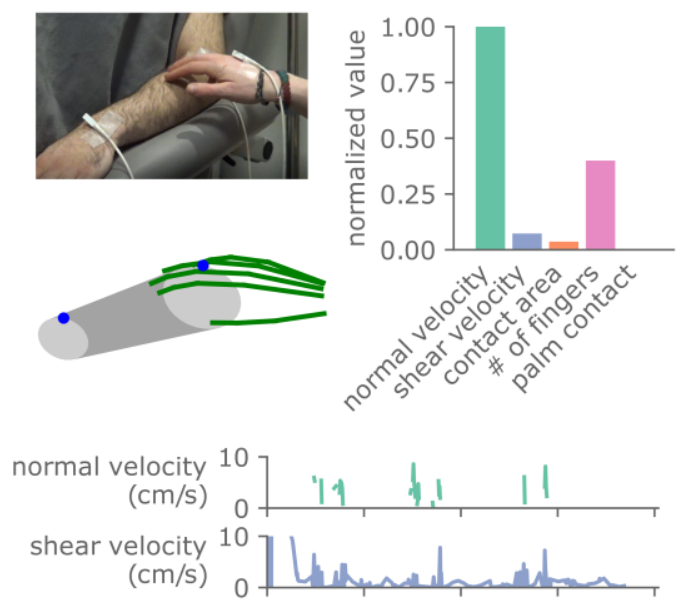
contact area 50

$\left(\mathrm{cm}^{2}\right)$
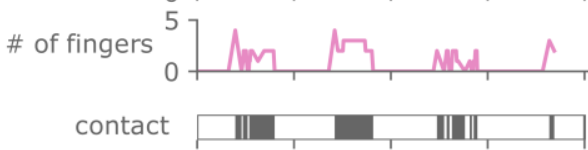

palm contact

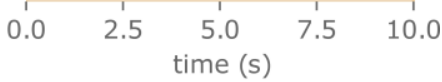

\section{gratitude}

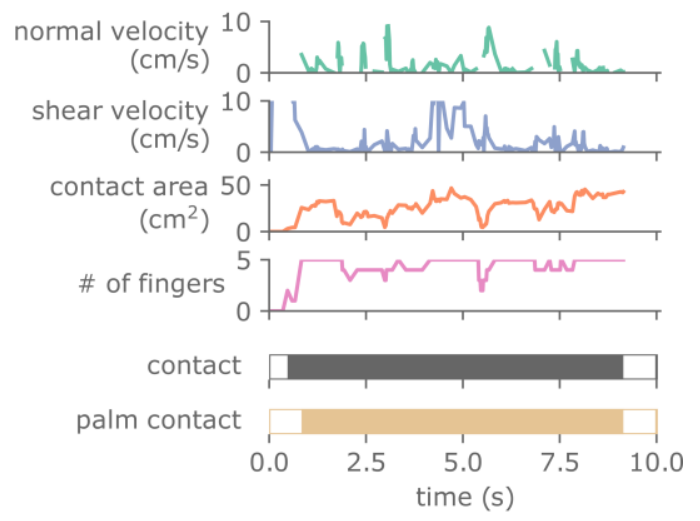

\section{happiness}

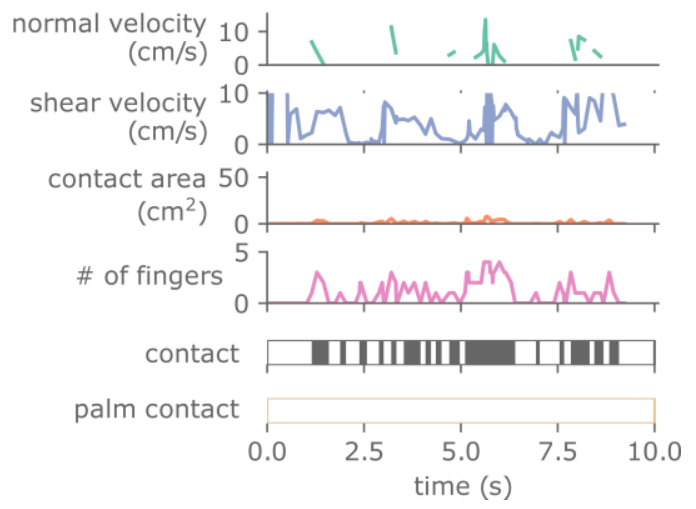


841 Figure S5. Time-slice of standardized gestures from hand-tracking system.

842

843 Movie S1. Video example of the standardized attention gesture.

844 Movie S2. Video example of the standardized love gesture.

845 Movie S3. Video example of the standardized happiness gesture.

846 Movie S4. Video example of the standardized calming gesture.

847 Movie S5. Video example of the standardized sadness gesture.

848 Movie S6. Video example of the standardized gratitude gesture. 
